ESAIM: M2AN 47 (2013) 1413-1432

DOI: $10.1051 / \mathrm{m} 2 \mathrm{an} / 2013074$
ESAIM: Mathematical Modelling and Numerical Analysis

www.esaim-m2an.org

\title{
A SIMPLE AND EFFICIENT SCHEME FOR PHASE FIELD CRYSTAL SIMULATION *
}

\author{
Matt Elsey ${ }^{1}$ AND Benedikt Wirth ${ }^{1}$
}

\begin{abstract}
We propose an unconditionally stable semi-implicit time discretization of the phase field crystal evolution. It is based on splitting the underlying energy into convex and concave parts and then performing $\mathrm{H}^{-1}$ gradient descent steps implicitly for the former and explicitly for the latter. The splitting is effected in such a way that the resulting equations are linear in each time step and allow an extremely simple implementation and efficient solution. We provide the associated stability and error analysis as well as numerical experiments to validate the method's efficiency.
\end{abstract}

Résumé. Une discrétisation semi-implicite et inconditionnellement stable est proposée pour l'évolution du modèle appelé «phase field crystal ». L'idée principale consiste à décomposer l'énergie correspondante en deux parties, l'une convexe et l'autre concave, puis à effectuer des pas de gradient $\mathrm{H}^{-1}$ pour lesquels on traite les deux parties implicitement et explicitement respectivement. La décomposition de l'énergie est choisie de façon à ce que l'équation resultante à résoudre à chaque pas de temps soit linéaire, ce qui conduit à un algorithme facile à implanter et efficace. Nous prouvons la stabilité et la convergence de l'algorithme puis nous présentons les résultats obtenus pour quelques expériences numériques.

Mathematics Subject Classification. 65M12, 74S25, 74N05, 74N20, 82C26.

Received March 19, 2012. Revised December 27, 2012.

Published online July 30, 2013.

\section{INTRODUCTION}

During the past few years, the so-called phase field crystal (PFC) method has become quite popular in physics $[4,5]$. It can be used to simulate the evolution of atomic crystals on time scales much longer than possible for molecular dynamics, while keeping much more detail than standard phase field models. In particular, it allows for the study of the evolution of lattice defects, the behavior of grain boundaries, the relation between elasticity and lattice distortion, and various further physical phenomena such as, for instance, epitaxial growth $[6,15]$ via simulation.

The method is based on a free energy, which in its simplest dimensionless form reads

$$
\mathcal{E}[u]=\int_{\Omega} \frac{1}{2}(\Delta u+u)^{2}-\frac{\delta}{2} u^{2}+\frac{1}{4} u^{4} \mathrm{~d} \mathbf{x} .
$$

\footnotetext{
Keywords and phrases. Phase field crystal, semi-implicit time discretization, convex-concave splitting.

* ME and BW gratefully acknowledge the support of NSF grant OISE-0967140 and of a Courant Instructorship, respectively.

1 Courant Institute of Mathematical Sciences, New York University, New York. \{melsey,benedikt.wirth\}@cims.nyu.edu
} 
Here, $\Omega \subset \mathbb{R}^{d}(d=1,2,3)$ is the domain occupied by some atomic material, $\delta$ has the interpretation of a dimensionless temperature, and the order parameter $u$ can be thought of as a probability density for the atom positions, whose average $\bar{u}=\frac{1}{|\Omega|} \int_{\Omega} u \mathrm{~d} \mathbf{x}$ is typically prescribed as a parameter. Depending on the parameters $\delta$ and $\bar{u}$, the energy $\mathcal{E}$ prefers different states, the most important being a constant-density liquid state and a periodic hexagonal crystalline state. Different variants of $\mathcal{E}$ can produce different crystal symmetries [13,14].

In physical simulations, the atomic density $u$ is evolved in time via an $\mathrm{H}^{-1}$ gradient flow (which conserves the average density $\bar{u}$ ) for the energy $\mathcal{E}$,

$$
u_{t}=\Delta\left((\Delta+1)^{2} u-\delta u+u^{3}\right) .
$$

Being sixth order parabolic, this partial differential equation is very stiff (explicit time stepping would by the CFL condition require the time step $\tau$ to be limited by the sixth power of the grid size, $h^{6}$, which is impracticable). Since one is typically interested in its long-time behavior, corresponding efficient, stable numerical schemes are indispensable. Note that the $\mathrm{L}^{2}$ gradient flow for the energy $\mathcal{E}$, known as the Swift-Hohenberg evolution, is also of physical interest. It is given by

$$
u_{t}=-(\Delta+1)^{2} u+\delta u-u^{3}
$$

and will in this paper be treated alongside with the phase field crystal equation.

Our proposed numerical scheme is closely related to the one proposed by Wise et al. [12], who base their scheme on a convex-concave splitting idea of Eyre [7]: If an energy $\mathcal{E}$ can be written as the difference of two convex energies $\mathcal{E}_{c}$ and $\mathcal{E}_{e}, \mathcal{E}=\mathcal{E}_{c}-\mathcal{E}_{e}$, then the time discretization

$$
\frac{u^{n+1}-u^{n}}{\tau}=-\nabla_{H} \mathcal{E}_{c}\left[u^{n+1}\right]+\nabla_{H} \mathcal{E}_{e}\left[u^{n}\right]
$$

of the gradient flow $u_{t}=-\nabla_{H} \mathcal{E}[u]$ is energy-stable, that is, it satisfies $\mathcal{E}\left[u^{n+1}\right] \leq \mathcal{E}\left[u^{n}\right]$ for all time steps $n$. Here, $\tau$ is the discrete time step, $u^{n}$ denotes the time-discrete approximation of $u(n \tau)$, and $\nabla_{H} \mathcal{E}$ denotes the gradient of an energy $\mathcal{E}$ with respect to the inner product on a Hilbert space $H$, defined by

$$
\left(\nabla_{H} \mathcal{E}[u], \theta\right)_{H}=\delta_{u} \mathcal{E}[u](\theta) \quad \forall \theta \in H,
$$

where the right-hand side is the Gâteaux derivative of $\mathcal{E}$ in a test direction $\theta$. Wise et al. decompose the phase field crystal energy into two convex energies according to

$$
\mathcal{E}_{c}[u]=\int_{\Omega} \frac{1}{2}(\Delta u)^{2}+\frac{1-\delta}{2} u^{2}+\frac{1}{4} u^{4} \mathrm{~d} \mathbf{x}, \quad \mathcal{E}_{e}[u]=\int_{\Omega}|\nabla u|^{2} \mathrm{~d} \mathbf{x}
$$

and then apply scheme (1.4) for $H \equiv \mathrm{H}^{-1}(\Omega)$, yielding an energy-stable time discretization of (1.2).

The disadvantage of the above approach is that each time step requires the solution of a nonlinear problem since the term $\frac{1}{4} u^{4}$ is part of the implicitly treated energy. We therefore aim to shift this term into the energy $\mathcal{E}_{e}$. However, in this case we also have to add an additional quadratic term to both $\mathcal{E}_{c}$ and $\mathcal{E}_{e}$ in order to make $\mathcal{E}_{e}$ sufficiently convex while respecting $\mathcal{E}=\mathcal{E}_{c}-\mathcal{E}_{e}$. In detail, for a sufficiently large constant $C>0$ and for the operator $L$ being either the identity $L \equiv 1$ or the gradient $L \equiv \nabla$, we propose to split the phase field crystal energy according to $\mathcal{E}=\mathcal{E}_{c}^{L}-\mathcal{E}_{\mathrm{e}}^{L}$ with

$$
\begin{aligned}
& \mathcal{E}_{c}^{L}[u]=\int_{\Omega} \frac{1}{2}(\Delta u+u)^{2}-\frac{\delta}{2} u^{2}+\frac{C}{2}|L u|^{2} \mathrm{~d} \mathbf{x}, \\
& \mathcal{E}_{\mathrm{e}}^{L}[u]=\int_{\Omega} \frac{C}{2}|L u|^{2}-\frac{1}{4} u^{4} \mathrm{~d} \mathbf{x}
\end{aligned}
$$

and then apply scheme (1.4) for $H \equiv \mathrm{H}^{-1}(\Omega)$, which turns out to yield a stable linear scheme.

This idea of adding and subtracting a term $\frac{C}{2}\|L u\|_{L^{2}(\Omega)}^{2}$ to a nonlinear energy $\mathcal{E}$ to obtain a stable linear time discretization is not new [7]. In different contexts, explicit time stepping schemes 
$\frac{1}{\tau}\left(u^{n+1}-u^{n}\right)=F\left[\Delta^{2} u^{n}, \Delta u^{n}, u^{n}\right]$ have been stabilized by adding and subtracting a higher order term $[8,9,11]$, $\frac{1}{\tau}\left(u^{n+1}-u^{n}\right)+C \Delta^{2} u^{n+1}=F\left[\Delta^{2} u^{n}, \Delta u^{n}, u^{n}\right]+C \Delta^{2} u^{n}$. Bertozzi et al. even stabilized a Cahn-Hilliard evolution with a lower order term [2]. However, stability in these works is only observed numerically and not proven.

Concerning numerical discretization of the phase field crystal evolution, a linear stable scheme has also been proposed by Cheng and Warren [3]. Besides the above-mentioned nonlinear convex-concave splitting scheme, Wise and coworkers also introduced a second order accurate two-step scheme for which they could prove the energy $\mathcal{E}$ to stay bounded. A different, promising approach is taken by Athreya et al. and Yeon et al. $[1,16]$, who derive differential equations for the amplitude of the order parameter $u$, valid in the regime where $u$ is approximately periodic, which can be solved on coarser grids and from whose solution $u$ can approximately be reconstructed.

The next section will introduce the proposed time discretization in detail and provide the corresponding stability and error analysis. The choice of the constant $C$ in (1.7) and (1.8) is discussed in Section 3. Section 4 introduces a spatial discretization and transfers the stability and error analysis to the fully discrete scheme. Finally, Section 5 shows various numerical experiments to demonstrate the practicability of the proposed scheme.

\section{An EFFiCIENT STABle time DiscRetizATion}

For simplicity, throughout this article we will assume $\Omega \subset \mathbb{R}^{n}$ to be a rectangular domain at whose boundary we impose periodic boundary conditions. In this section, we prove the stability and convergence of the proposed scheme.

\subsection{Stable convex-concave splitting}

For a sufficiently large constant $C>0$ and for the operator $L$ being either the identity $L \equiv 1$ or the gradient $L \equiv \nabla$, we propose to split the phase field crystal energy according to $\mathcal{E}=\mathcal{E}_{c}^{L}-\mathcal{E}_{\mathrm{e}}^{L}$ with

$$
\begin{aligned}
& \mathcal{E}_{c}^{L}[u]=\int_{\Omega} \frac{1}{2}(\Delta u+u)^{2}-\frac{\delta}{2} u^{2}+\frac{C}{2}|L u|^{2} \mathrm{~d} \mathbf{x}, \\
& \mathcal{E}_{\mathrm{e}}^{L}[u]=\int_{\Omega} \frac{C}{2}|L u|^{2}-\frac{1}{4} u^{4} \mathrm{~d} \mathbf{x} .
\end{aligned}
$$

This splitting naturally leads to the semi-implicit time discretization of the Swift-Hohenberg or the phase field crystal evolution given by (1.4). Its strong form, for the choices (2.1) and (2.2), thus reads

$$
\frac{u^{n+1}-u^{n}}{\tau}=-(\Delta+1)^{2} u^{n+1}+\delta u^{n+1}-C L^{*} L\left(u^{n+1}-u^{n}\right)-\left(u^{n}\right)^{3}
$$

for the Swift-Hohenberg scheme and

$$
\frac{u^{n+1}-u^{n}}{\tau}=\Delta\left[(\Delta+1)^{2} u^{n+1}-\delta u^{n+1}+C L^{*} L\left(u^{n+1}-u^{n}\right)+\left(u^{n}\right)^{3}\right]
$$

for the phase field crystal evolution, where either $L^{*} L \equiv 1$ or $L^{*} L \equiv-\Delta$.

The above scheme satisfies many desirable properties. First of all, just as in the continuous case, the timediscrete phase field crystal evolution conserves the average value $\bar{u}$. This is readily seen by integrating both sides of (2.4) over $\Omega$ and applying the divergence theorem together with the periodic boundary conditions. Also, for $C$ chosen sufficiently large, each time step is uniquely solvable for $u^{n+1}$, since (2.3) and (2.4) are equivalent to solving the strictly convex minimization problem

$$
u^{n+1}=\underset{u}{\operatorname{argmin}} \frac{\left\|u-u^{n}\right\|_{\mathrm{H}}^{2}}{2 \tau}+\mathcal{E}_{c}^{L}[u]-\delta_{u} \mathcal{E}_{\mathrm{e}}^{L}\left[u^{n}\right](u),
$$

which is coercive (the strict convexity and coercivity for $C \geq 2$ are readily seen when expanding all squares in the above energy). Here, $H \equiv \mathrm{L}^{2}(\Omega)$ for the Swift-Hohenberg scheme, and $H \equiv \mathrm{H}^{-1}(\Omega)$ for the phase field crystal 
evolution. Apparently, the scheme is first order consistent and in each time step only requires the solution of a constant coefficient linear problem, which allows highly efficient implementations (e.g. via Fourier transforms, see Sect. 5). Moreover, the linear system to be solved is the same in every time step, which makes the scheme extremely simple to implement.

For $C$ large enough, the proposed scheme is $\mathrm{L}^{\infty}$-stable and decreases the phase field crystal energy in every step, as shown by the following theorem.

Theorem 2.1 (Stability). Assume $\delta<1$. For any $u^{0}: \Omega \rightarrow \mathbb{R}$ with finite energy $\mathcal{E}$ there exists a $C>0$ such that the schemes (2.3) (for $L \equiv 1$ ) and (2.4) (for $L \equiv 1, \nabla$ ) are stable for any $\tau>0$ in the sense

$$
\begin{aligned}
& \mathcal{E}\left[u^{n+1}\right] \leq \mathcal{E}\left[u^{n}\right] \quad \forall n \in \mathbb{N}, \\
& \exists U>0:\left\|u^{n}\right\|_{L^{\infty}(\Omega)} \leq U \forall n \in \mathbb{N} .
\end{aligned}
$$

Proof. Let us abbreviate $\mathcal{E}\left[u^{0}\right]=\hat{\mathcal{E}}$. The same argument as in [12] leads to a bound

$$
\left\|u^{0}\right\|_{L^{\infty}(\Omega)} \leq \sqrt{\frac{\hat{\mathcal{E}}+|\Omega| / 4}{\gamma}}=: U
$$

for some $\gamma>0$ independent of $u^{0}$, where without loss of generality we assume $U \geq 1$. Indeed, using $\frac{1}{4}\|u\|_{\mathrm{L}^{4}(\Omega)}^{4} \geq$ $\frac{1}{2}\|u\|_{\mathrm{L}^{2}(\Omega)}^{2}-\frac{|\Omega|}{4}$ and $\|\nabla u\|_{\mathrm{L}^{2}(\Omega)}^{2}=-(u, \Delta u)_{\mathrm{L}^{2}(\Omega)} \leq \frac{1}{2 \beta}\|u\|_{\mathrm{L}^{2}(\Omega)}^{2}+\frac{\beta}{2}\|\Delta u\|_{\mathrm{L}^{2}(\Omega)}^{2}$ for any $\beta>0$ we obtain

$$
\begin{aligned}
\mathcal{E}[u] & =\frac{1}{4}\|u\|_{\mathrm{L}^{4}(\Omega)}^{4}+\frac{1-\delta}{2}\|u\|_{\mathrm{L}^{2}(\Omega)}^{2}-\|\nabla u\|_{\mathrm{L}^{2}(\Omega)}^{2}+\frac{1}{2}\|\Delta u\|_{\mathrm{L}^{2}(\Omega)}^{2} \\
& \geq \frac{2-\delta-\frac{1}{\beta}}{2}\|u\|_{\mathrm{L}^{2}(\Omega)}^{2}+\frac{1-\beta}{2}\|\Delta u\|_{\mathrm{L}^{2}(\Omega)}^{2}-\frac{|\Omega|}{4} \\
& \geq \frac{3}{2} \eta\left(\|u\|_{\mathrm{L}^{2}(\Omega)}^{2}+\|\Delta u\|_{\mathrm{L}^{2}(\Omega)}^{2}\right)-\frac{|\Omega|}{4}
\end{aligned}
$$

for $\eta=\min \left(\frac{2-\delta-\frac{1}{\beta}}{3}, \frac{1-\beta}{3}\right)>0$ (take, e.g., $\left.\beta=\frac{2}{3-\delta}\right)$. Hence,

$$
\begin{aligned}
\mathcal{E}[u]+\frac{|\Omega|}{4} & \geq \eta\left(\|u\|_{\mathrm{L}^{2}(\Omega)}^{2}+\|\Delta u\|_{\mathrm{L}^{2}(\Omega)}^{2}+\frac{1}{2}\left(\|u\|_{\mathrm{L}^{2}(\Omega)}^{2}+\|\Delta u\|_{\mathrm{L}^{2}(\Omega)}^{2}\right)\right) \\
& \geq \eta\left(\|u\|_{\mathrm{L}^{2}(\Omega)}^{2}+\|\nabla u\|_{\mathrm{L}^{2}(\Omega)}^{2}+\|\Delta u\|_{\mathrm{L}^{2}(\Omega)}^{2}\right) \geq \gamma\|u\|_{\mathrm{L}^{\infty}(\Omega)}^{2}
\end{aligned}
$$

for some $\gamma>0$ by Sobolev embedding. We will show that for an appropriate choice of $C,\left\|u^{n}\right\|_{\mathrm{L}^{\infty}(\Omega)} \leq U$ and $\mathcal{E}\left[u^{n}\right] \leq \hat{\mathcal{E}}$ for all $n \in \mathbb{N}$.

Choose

$$
C>\max \left(\delta, 2,3 U^{2}, \frac{3}{\nu} U^{2}, 3 \frac{\hat{\mathcal{E}}+\frac{5}{4}|\Omega| U^{4}}{\gamma}, \frac{3}{\nu} \frac{\hat{\mathcal{E}}+\frac{5}{4}|\Omega| U^{4}}{\gamma}\right),
$$

where $\nu$ is the constant from the Poincare inequality, satisfying $\|\nabla u\|_{\mathrm{L}^{2}(\Omega)}^{2} \geq \nu\|u-\bar{u}\|_{\mathrm{L}^{2}(\Omega)}^{2}$ with $\bar{u}$ being the average of $u$. We will prove the theorem by induction on $n \in \mathbb{N}$, so let us assume $\mathcal{E}\left[u^{n}\right] \leq \hat{\mathcal{E}}$ and $\left\|u^{n}\right\|_{L^{\infty}(\Omega)} \leq U$ to hold. We know that $u^{n+1} \in N$, where $N=\left\{u \in \mathrm{L}^{2}(\Omega) \mid \int_{\Omega} u \mathrm{~d} \mathbf{x}=\int_{\Omega} u^{0} \mathrm{~d} \mathbf{x}\right\}$ for scheme (2.4) and $N=\mathrm{L}^{2}(\Omega)$ for scheme (2.3).

First note that $\mathcal{E}_{c}^{L}$ is a convex functional. Let us introduce

$$
\begin{aligned}
& \tilde{\mathcal{E}}_{\mathrm{e}}^{L}[u]=\int_{\Omega} \frac{C}{2}|L u|^{2}-f(u) \mathrm{d} \mathbf{x}, \quad \text { where } \\
& f(u)= \begin{cases}\frac{1}{4} u^{4}, & |u| \leq U, \\
\frac{3}{2} U^{2} u^{2}-2 U^{3}|u|+\frac{3}{4} U^{4}, & \text { else. }\end{cases}
\end{aligned}
$$


$\tilde{\mathcal{E}}_{\mathrm{e}}^{L}$ is convex on $N$ : Indeed, its second Gâteaux derivative in some test direction $\theta$ satisfies

$$
\delta_{u}^{2} \tilde{\mathcal{E}}_{\mathrm{e}}^{L}[u](\theta, \theta)=C\|L \theta\|_{\mathrm{L}^{2}(\Omega)}^{2}-\int_{\Omega} 3 \min \left(u^{2}, U^{2}\right) \theta^{2} \mathrm{~d} x \geq C\|L \theta\|_{\mathrm{L}^{2}(\Omega)}^{2}-3 U^{2}\|\theta\|_{\mathrm{L}^{2}(\Omega)}^{2} .
$$

In the case $L \equiv 1$ this is clearly non-negative. The case $L \equiv \nabla$ is only considered for the phase field crystal evolution. In that case, since the test functions may not lead outside of $N$, we have $\int_{\Omega} \theta \mathrm{d} \mathbf{x}=0$. Thus, the non-negativity of the second Gâteaux derivative follows by Poincaré's inequality. By the analogous argument we obtain that $\mathcal{E}_{\mathrm{e}}^{L}$ is convex on $\left\{u \in N \mid\|u\|_{\mathrm{L}^{\infty}(\Omega)}^{2} \leq \frac{C}{3} \min (1, \nu)\right\}$.

We now employ the classical convex-concave splitting argument: By the convexity of $\mathcal{E}_{c}^{L}$ and $\tilde{\mathcal{E}}_{\mathrm{e}}^{L}$,

$$
\begin{aligned}
\mathcal{E}_{c}^{L}\left[u^{n+1}\right]-\tilde{\mathcal{E}}_{\mathrm{e}}^{L}\left[u^{n+1}\right] & \leq \mathcal{E}_{c}^{L}\left[u^{n}\right]-\tilde{\mathcal{E}}_{\mathrm{e}}^{L}\left[u^{n}\right]+\left(\delta_{u} \mathcal{E}_{c}^{L}\left[u^{n+1}\right]-\delta_{u} \tilde{\mathcal{E}}_{\mathrm{e}}^{L}\left[u^{n}\right]\right)\left(u^{n+1}-u^{n}\right) \\
& =\mathcal{E}_{c}^{L}\left[u^{n}\right]-\mathcal{E}_{\mathrm{e}}^{L}\left[u^{n}\right]+\left(\delta_{u} \mathcal{E}_{c}^{L}\left[u^{n+1}\right]-\delta_{u} \mathcal{E}_{\mathrm{e}}^{L}\left[u^{n}\right]\right)\left(u^{n+1}-u^{n}\right) \\
& =\mathcal{E}\left[u^{n}\right]-\frac{1}{\tau}\left(u^{n+1}-u^{n}, u^{n+1}-u^{n}\right)_{H} \leq \mathcal{E}\left[u^{n}\right] \leq \hat{\mathcal{E}},
\end{aligned}
$$

where $H \equiv \mathrm{L}^{2}(\Omega)$ for the Swift-Hohenberg scheme, and $H \equiv \mathrm{H}^{-1}(\Omega)$ for the phase field crystal evoluation. However, this implies $\left\|u^{n+1}\right\|_{L^{\infty}(\Omega)}^{2} \leq \frac{\hat{\mathcal{E}}+\frac{5}{4}|\Omega| U^{4}}{\gamma} \leq \frac{C}{3} \min (1, \nu)$, since for any $u$ we have

$$
\begin{aligned}
\hat{\mathcal{E}} \geq \mathcal{E}_{c}^{L}[u]-\tilde{\mathcal{E}}_{\mathrm{e}}^{L}[u] & =\frac{1}{2}\|\Delta u+u\|_{\mathrm{L}^{2}(\Omega)}^{2}+\int_{\Omega} f(u)-\frac{\delta}{2} u^{2} \mathrm{~d} x \\
& \geq \frac{1}{2}\|\Delta u+u\|_{\mathrm{L}^{2}(\Omega)}^{2}+\frac{U^{2}}{2}\|u\|_{\mathrm{L}^{2}(\Omega)}^{2}-\frac{5}{4}|\Omega| U^{4} \\
& \geq \gamma\|u\|_{\mathrm{L}^{\infty}(\Omega)}^{2}-\frac{5}{4}|\Omega| U^{4}
\end{aligned}
$$

using the same estimates as earlier. Now, since $\mathcal{E}_{\mathrm{e}}^{L}$ is convex on $\left\{u \in N \mid\|u\|_{\mathrm{L}^{\infty}(\Omega)}^{2} \leq \frac{C}{3} \min (1, \nu)\right\}$, we may again apply the classical convex-concave splitting argument, this time to $\mathcal{E}_{c}^{L}-\mathcal{E}_{\mathrm{e}}^{L}$, which yields

$$
\mathcal{E}\left[u^{n+1}\right]=\mathcal{E}_{c}^{L}\left[u^{n+1}\right]-\mathcal{E}_{\mathrm{e}}^{L}\left[u^{n+1}\right] \leq \mathcal{E}_{c}^{L}\left[u^{n}\right]-\mathcal{E}_{\mathrm{e}}^{L}\left[u^{n}\right]+\left(\delta_{u} \mathcal{E}_{c}^{L}\left[u^{n+1}\right]-\delta_{u} \mathcal{E}_{\mathrm{e}}^{L}\left[u^{n}\right]\right)\left(u^{n+1}-u^{n}\right) \leq \mathcal{E}\left[u^{n}\right] \leq \hat{\mathcal{E}}
$$

and thus also $\left\|u^{n+1}\right\|_{\mathrm{L}^{\infty}(\Omega)} \leq U$.

Remark 2.2. The condition $\delta<1$ can be removed by slightly improving the derivation of the $\mathrm{L}^{\infty}$-bound from $\mathcal{E}[u] \leq \hat{\mathcal{E}}$.

\subsection{Convergence of the scheme}

The stability and first order consistency imply first order convergence. The argument is similar to the analysis for the scheme in [12], nevertheless we will provide a compact version of the proof for the sake of completeness. Quite naturally, it is essential for the convergence that $C$ does not depend on $\tau$ (as proven by the previous theorem) so that during the following analysis one should think of $C$ as an $O(1)$ constant.

Theorem 2.3 (Error estimate). Suppose the Swift-Hohenberg (or phase field crystal) equation is solved by a smooth spatially periodic function $u:[0, T] \times \Omega \rightarrow \mathbb{R}$ for some $T \in(0, \infty)$, and denote the solution to $(2.3)$ (or (2.4)) by $u^{n}, n=0,1, \ldots$, where $u^{0}=u(0)$ and $C$ is chosen according to Theorem 2.1. Then, there exists a constant $K>0$ independent of $\tau$ such that (for $\tau$ small enough)

$$
\left\|u(n \tau)-u^{n}\right\|_{L^{2}(\Omega)} \leq K \tau
$$

for all $n$ with $n \tau \leq T$. 
Proof. We show the argument for the phase field crystal equation (the treatment of the Swift-Hohenberg equation only requires obvious adaptions). Let us abbreviate $t^{n}=n \tau$ and $\mathrm{e}^{n}=u\left(t^{n}\right)-u^{n}$. The time-continuous solution satisfies

$$
\frac{u\left(t^{n+1}\right)-u\left(t^{n}\right)}{\tau}=\Delta\left[\left((\Delta+1)^{2}-\delta\right) u\left(t^{n+1}\right)+C L^{*} L\left(u\left(t^{n+1}\right)-u\left(t^{n}\right)\right)+u\left(t^{n}\right)^{3}\right]+\rho^{n+1},
$$

where by a second order Taylor expansion about $t^{n+1}$ the truncation error $\rho^{n+1}$ satisfies

$$
\left|\rho^{n+1}\right| \leq \tau\left(\frac{1}{2}\|u\|_{\mathrm{C}^{2}\left([0, T], \mathrm{C}^{0}(\Omega)\right)}+C\|u\|_{\mathrm{C}^{1}\left([0, T], \mathrm{C}^{a}(\Omega)\right)}+\|u\|_{\mathrm{C}^{1}\left([0, T], \mathrm{C}^{2}(\Omega)\right)}^{3}\right)=: \tilde{K} \tau
$$

with $a=2$ for $L \equiv 1$ and $a=4$ for $L \equiv \nabla$. Subtracting (2.4), we obtain

$$
\frac{\mathrm{e}^{n+1}-\mathrm{e}^{n}}{\tau}=\Delta\left[\left((\Delta+1)^{2}-\delta\right) \mathrm{e}^{n+1}+C L^{*} L\left(\mathrm{e}^{n+1}-\mathrm{e}^{n}\right)+u\left(t^{n}\right)^{3}-\left(u^{n}\right)^{3}\right]+\rho^{n+1} .
$$

Testing with $\tau \mathrm{e}^{n+1}$ and integrating some terms by parts, we arrive at

$$
\begin{aligned}
\left\|\mathrm{e}^{n+1}\right\|^{2}-\left(\mathrm{e}^{n}, \mathrm{e}^{n+1}\right)= & \tau\left[-\left\|\nabla \Delta \mathrm{e}^{n+1}\right\|^{2}+2\left\|\Delta \mathrm{e}^{n+1}\right\|^{2}-(1-\delta)\left\|\nabla \mathrm{e}^{n+1}\right\|^{2}\right. \\
& \left.-C\left\|L^{*} \nabla \mathrm{e}^{n+1}\right\|^{2}+C\left(L^{*} \nabla \mathrm{e}^{n}, L^{*} \nabla \mathrm{e}^{n+1}\right)+\left(u\left(t^{n}\right)^{3}-\left(u^{n}\right)^{3}, \Delta \mathrm{e}^{n+1}\right)+\left(\rho^{n+1}, \mathrm{e}^{n+1}\right)\right],
\end{aligned}
$$

where for simplicity we wrote $\|\cdot\|$ for the $\mathrm{L}^{2}(\Omega)$-norm and $(\cdot, \cdot)$ for the corresponding inner product. We now apply Young's inequality $(v, w) \leq \frac{1}{2 \varepsilon}\|v\|^{2}+\frac{\varepsilon}{2}\|w\|^{2}$ (with appropriate $\varepsilon$ ) to all inner products, which yields

$$
\begin{aligned}
\frac{\left\|\mathrm{e}^{n+1}\right\|^{2}-\left\|\mathrm{e}^{n}\right\|^{2}}{2} \leq & \tau\left[-\left\|\nabla \Delta \mathrm{e}^{n+1}\right\|^{2}+\frac{5}{2}\left\|\Delta \mathrm{e}^{n+1}\right\|^{2}-(1-\delta)\left\|\nabla \mathrm{e}^{n+1}\right\|^{2}\right. \\
& -C\left(1-\frac{1}{2 \alpha}\right)\left\|L^{*} \nabla \mathrm{e}^{n+1}\right\|^{2}+\frac{C \alpha}{2}\left\|L^{*} \nabla \mathrm{e}^{n}\right\|^{2} \\
& \left.+\frac{1}{2}\left\|u\left(t^{n}\right)^{3}-\left(u^{n}\right)^{3}\right\|^{2}+\frac{1}{2}\left\|\rho^{n+1}\right\|^{2}+\frac{1}{2}\left\|\mathrm{e}^{n+1}\right\|^{2}\right]
\end{aligned}
$$

for any $\alpha>0$. Since by Theorem 2.1 both $u\left(t^{n}\right)$ and $u^{n}$ are bounded in $\mathrm{L}^{\infty}$ by some constant $U$, we have $\left\|u\left(t^{n}\right)^{3}-\left(u^{n}\right)^{3}\right\|^{2} \leq \hat{K}\left\|\mathrm{e}^{n}\right\|^{2}$, where $\sqrt{\hat{K}}$ is the Lipschitz constant of $(\cdot)^{3}$ on $[-U, U]$. Furthermore, $\frac{5}{2}\left\|\Delta \mathrm{e}^{n+1}\right\|^{2} \leq$ $\frac{125}{54}\left\|\mathrm{e}^{n+1}\right\|^{2}+\left\|\nabla \Delta \mathrm{e}^{n+1}\right\|^{2}$ due to

$\|\Delta v\|^{2}=-(\nabla v, \nabla \Delta v) \leq \frac{1}{2 \varepsilon}\|\nabla v\|^{2}+\frac{\varepsilon}{2}\|\nabla \Delta v\|^{2}=-\frac{1}{2 \varepsilon}(v, \Delta v)+\frac{\varepsilon}{2}\|\nabla \Delta v\|^{2} \leq \frac{1}{4 \varepsilon^{2}}\|v\|^{2}+\frac{1}{4}\|\Delta v\|^{2}+\frac{\varepsilon}{2}\|\nabla \Delta v\|^{2}$.

Applying these estimates, we obtain

$$
\begin{aligned}
\frac{\left\|\mathrm{e}^{n+1}\right\|^{2}-\left\|\mathrm{e}^{n}\right\|^{2}}{2} \leq \tau\left[-(1-\delta)\left\|\nabla \mathrm{e}^{n+1}\right\|^{2}-C\left(1-\frac{1}{2 \alpha}\right)\left\|L^{*} \nabla \mathrm{e}^{n+1}\right\|^{2}\right. & \\
& \left.\quad+\frac{C \alpha}{2}\left\|L^{*} \nabla \mathrm{e}^{n}\right\|^{2}+\frac{\hat{K}}{2}\left\|\mathrm{e}^{n}\right\|^{2}+\frac{|\Omega| \tilde{K}^{2}}{2} \tau^{2}+\frac{76}{27}\left\|\mathrm{e}^{n+1}\right\|^{2}\right]
\end{aligned}
$$

and thus by induction (choosing $\alpha$ such that $\alpha=\left(2-\frac{1}{\alpha}\right) \frac{1+\hat{K} \tau}{1-\frac{152}{27} \tau}$ )

$$
\begin{aligned}
\left\|\mathrm{e}^{n+1}\right\|^{2} \leq & \frac{(1+\hat{K} \tau)\left\|\mathrm{e}^{n}\right\|^{2}+|\Omega| \tilde{K}^{2} \tau^{3}+C \tau\left(\alpha\left\|L^{*} \nabla \mathrm{e}^{n}\right\|^{2}-\left(2-\frac{1}{\alpha}\right)\left\|L^{*} \nabla \mathrm{e}^{n+1}\right\|^{2}\right)}{1-\frac{152}{27} \tau} \\
\leq & \left(\frac{1+\hat{K} \tau}{1-\frac{152}{27} \tau}\right)^{n+1}\left\|\mathrm{e}^{0}\right\|^{2}+\frac{|\Omega| \tilde{K}^{2} \tau^{3}}{1-\frac{152}{27} \tau} \sum_{j=0}^{n}\left(\frac{1+\hat{K} \tau}{1-\frac{152}{27} \tau}\right)^{j} \\
& +\frac{C \tau}{1-\frac{152}{27} \tau}\left(\alpha\left(\frac{1+\hat{K} \tau}{1-\frac{152}{27} \tau}\right)^{n}\left\|L^{*} \nabla \mathrm{e}^{0}\right\|^{2}-\left(2-\frac{1}{\alpha}\right)\left\|L^{*} \nabla \mathrm{e}^{n+1}\right\|^{2}\right) \\
\leq & \frac{|\Omega| \tilde{K}^{2} \tau^{3}}{1-\frac{152}{27} \tau} \sum_{j=0}^{n}\left(\frac{1+\hat{K} \tau}{1-\frac{152}{27} \tau}\right)^{j}=|\Omega| \tilde{K}^{2} \tau^{2} \frac{\left(\frac{1+\hat{K} \tau}{1-\frac{152}{2} \tau}\right)^{n+1}-1}{\hat{K}+\frac{152}{27}} .
\end{aligned}
$$


Due to $t^{n+1} \leq T$ we have $\left(\frac{1+\hat{K} \tau}{1-\frac{152}{27} \tau}\right)^{n+1} \leq\left(\frac{1+\frac{\hat{K} T}{n+1}}{1-\frac{152 T}{27(n+1)}}\right)^{n+1} \rightarrow_{n \rightarrow \infty} \exp \left(T\left(\hat{K}+\frac{152}{27}\right)\right)$ so that the factor is bounded for $\tau$ small enough (or equivalently, $n$ large enough), which concludes the proof.

\section{Guidelines For Choosing $C$}

In this section we attempt to provide some understanding of the effect of the choice of $C$. First, we consider the Swift-Hohenberg evolution, where it can be immediately seen that choosing a large $C$ slows the evolution; indeed, the effective time step is shown to be proportional to $C^{-1}$ as $\tau \rightarrow \infty$. Here we also present a simple condition on $C$ to guarantee the energy stability of the Swift-Hohenberg evolution. Next, we proceed to the more complicated phase field crystal evolution. We emulate a calculation of Cheng and Warren [3] to understand the effect of the choice of $C$ on the phase field evolution, finding that the maximum effective time step is again proportional to $C^{-1}$ as $\tau \rightarrow \infty$. For this reason, it is preferable to choose $C$ as small as possible while still maintaining the unconditional stability of the algorithm. We conclude this section by presenting some heuristic arguments to motivate an expression for the choice of $C$ which seems to be sufficient to give stable numerical solutions for a wide variety of problems in the context of the phase field crystal evolution.

\subsection{Swift-Hohenberg evolution}

The constant $C$, which stabilizes the time stepping scheme, should be chosen as small as possible, not only for accuracy reasons, but primarily because a larger $C$ effects a slower evolution. This is particularly easy to see for the Swift-Hohenberg case: The scheme (2.3) (with $L \equiv 1$ ) is equivalent to the time discretization

$$
\frac{u^{n+1}-u^{n}}{\tilde{\tau}}=-(\Delta+1)^{2} u^{n+1}+\delta u^{n+1}-\left(u^{n}\right)^{3}
$$

with time step $\tilde{\tau}=\frac{\tau}{1+C \tau}$. For the phase field crystal equation the relation is not as simple, but qualitatively similar, and will be discussed later in this section. It therefore appears beneficial to understand what phenomena limit the value of $C$. The proof of Theorem 2.1 provides a lower bound just in terms of the initial energy $\mathcal{E}\left[u^{0}\right]$, but this bound is based on a number of non-sharp estimates and thus must be quite pessimistic. The matter seems more accessible for the Swift-Hohenberg discretization, for which we will provide a brief analysis in the following.

Theorem 3.1 (Stability of (3.1)). If $C>0$ is chosen such that the operator $A^{\tilde{C}}=\left[1-\frac{\delta}{\tilde{C}}+\frac{(\Delta+1)^{2}}{\tilde{C}}\right]^{-1}$ : $\mathrm{L}^{\infty}(\Omega) \rightarrow \mathrm{L}^{\infty}(\Omega)$ has norm $\left\|A^{\tilde{C}}\right\|_{\mathrm{L}^{\infty}} \leq \frac{3}{2}$ for all $\tilde{C} \geq C$, then the discretization (3.1) satisfies the following stability properties: If $\left\|u^{0}\right\|_{L^{\infty}(\Omega)} \leq \sqrt{\frac{C}{3}}$ and $\tilde{\tau}<\frac{1}{C}$, then

$$
\begin{aligned}
& \mathcal{E}\left[u^{n+1}\right] \leq \mathcal{E}\left[u^{n}\right] \quad \forall n \in \mathbb{N}, \\
& \left\|u^{n}\right\|_{L^{\infty}(\Omega)} \leq 1 / \sqrt{3 \tilde{\tau}} \quad \forall n \in \mathbb{N} .
\end{aligned}
$$

Proof. Iteration (3.1) can be expressed as $u^{n+1}=A^{\frac{1}{\tau}}\left(u^{n}-\tilde{\tau}\left(u^{n}\right)^{3}\right)$. Obviously, $\left\|u^{n}\right\|_{L^{\infty}(\Omega)} \leq 1 / \sqrt{3 \tilde{\tau}}$ implies $\left\|u^{n+1}\right\|_{\mathrm{L}^{\infty}(\Omega)} \leq\left\|A^{\frac{1}{\tilde{\tau}}}\right\|_{\mathrm{L}^{\infty}}\left\|u^{n}-\tilde{\tau}\left(u^{n}\right)^{3}\right\|_{\mathrm{L}^{\infty}(\Omega)} \leq \frac{3}{2} \cdot \frac{2}{3 \sqrt{3 \tilde{\tau}}}$, which upon induction proves the second inequality.

For the first inequality we note that (3.1) is equivalent to $0=-\delta_{u} \mathcal{E}_{c}^{1}\left[u^{n+1}\right]+\delta_{u} \mathcal{E}_{\mathrm{e}}^{1}\left[u^{n}\right]$ for the parameter choice $C=\frac{1}{\tilde{\tau}}$. Both $\mathcal{E}_{c}^{1}$ and $\mathcal{E}_{\mathrm{e}}^{1}$ are convex on $\left\{u \in \mathrm{L}^{\infty}(\Omega) \mid\|u\|_{\mathrm{L}^{\infty}(\Omega)} \leq 1 / \sqrt{3 \tilde{\tau}}\right\}=: M$, and by the previous part we may assume $u^{n}, u^{n+1} \in M$. Hence, by the usual convex-concave splitting argument,

$$
\begin{aligned}
\mathcal{E}\left[u^{n+1}\right] & =\mathcal{E}_{c}^{1}\left[u^{n+1}\right]-\mathcal{E}_{\mathrm{e}}^{1}\left[u^{n+1}\right] \\
& \leq \mathcal{E}_{c}^{1}\left[u^{n}\right]-\mathcal{E}_{\mathrm{e}}^{1}\left[u^{n}\right]+\left(\delta_{u} \mathcal{E}_{c}^{1}\left[u^{n+1}\right]-\delta_{u} \mathcal{E}_{\mathrm{e}}^{1}\left[u^{n}\right]\right)\left(u^{n+1}-u^{n}\right) \\
& =\mathcal{E}_{c}^{1}\left[u^{n}\right]-\mathcal{E}_{\mathrm{e}}^{1}\left[u^{n}\right]=\mathcal{E}\left[u^{n}\right] .
\end{aligned}
$$


Remark 3.2. If $C$ is chosen such that only $\left\|A^{\tilde{C}}\right\|_{\mathrm{L}^{\infty}} \leq 3$ for all $\tilde{C} \geq C$, then by the same proof $\left\|u^{0}\right\|_{\mathrm{L}^{\infty}(\Omega)} \leq$ $2 \sqrt{C / 3}$ and $\tilde{\tau}<\frac{1}{C}$ imply $\left\|u^{n}\right\|_{L^{\infty}(\Omega)} \leq 2 / \sqrt{3 \tilde{\tau}}$ for all $n \in \mathbb{N}$, however, we can no longer guarantee $\mathcal{E}\left[u^{n+1}\right] \leq$ $\mathcal{E}\left[u^{n}\right]$.

The above implies that unconditional stability of scheme (2.3) (given appropriate input data) is already guaranteed if we choose $C$ such that all $\tilde{C}>C$ satisfy $\left\|A^{\tilde{C}}\right\|_{\mathrm{L}^{\infty}} \leq \frac{3}{2}$ (for $\mathrm{L}^{\infty}$ and energy stability) or even only $\left\|A^{\tilde{C}}\right\|_{\mathrm{L}^{\infty}} \leq 3$ (for $\mathrm{L}^{\infty}$ stability). It turns out that $\left\|A^{\tilde{C}}\right\|_{\mathrm{L}^{\infty}} \rightarrow \tilde{C} \rightarrow \infty\left\|\mathcal{F}\left[1 /\left(1+|\cdot|^{4}\right)\right]\right\|_{L^{1}\left(\mathbb{R}^{d}\right)}=$ $\left\|\mathcal{F}^{-1}\left[1 /\left(1+|\cdot|^{4}\right)\right]\right\|_{\mathrm{L}^{1}\left(\mathbb{R}^{d}\right)}$ (see Appendix 5.6), where $\mathcal{F}$ denotes the Fourier transform on $\mathbb{R}^{d}$. For $d=1,2,3$, this number is smaller than $\frac{3}{2}$ so that an appropriate $C$ can indeed be found.

Note that the proof of Theorem 2.1 only provides sufficient lower bounds on $C$, but no information on whether these bounds are good or on what type of initial data would lead to instabilities for too small $C$. However, the analysis of the operator $A^{C}$ can provide some intuition: The proof of Lemma A.1 in the appendix shows that the operator $A^{C}$ can be expressed as a convolution with a continuous function $h^{C}$. Hence, $A^{C}$ increases the $\mathrm{L}^{\infty}$ norm most if it is applied to $\operatorname{sign}\left(h^{C}(-\cdot)\right): \Omega \rightarrow\{-1,1\}$, which almost looks like a periodic oscillation between -1 and 1. Expressed differently, the initial data $u^{0}=\operatorname{sign}\left(h^{C}(-\cdot)\right)$ is most critical with respect to $\mathrm{L}^{\infty}$ stability; furthermore one can observe that discontinuous step functions in general are quite critical. This effect is similar to the Gibbs phenomenon. Indeed, $A^{C}$ acts like a low pass filter which damps all frequencies larger than $\sqrt[4]{C}$. Hence, application of $A^{C}$ to a step function results in local overshoots near the discontinuity and thus in an increased $\mathrm{L}^{\infty}$ norm. For smooth initial data on the other hand, the operator $A^{C}$ is rather well-behaved so that for smooth initial data $C$ can be chosen smaller and one need not require $\left\|A^{C}\right\|_{\mathrm{L}^{\infty}} \leq \frac{3}{2}$.

\subsection{Phase field crystal evolution}

Though the phase field crystal model is more difficult to analyze than Swift-Hohenberg, we present some heuristic calculations which suggest that the situation with regard to the choice of $C$ is similar in this case. As suggested by the work of Cheng and Warren [3], we calculate the Fourier space "effective time step", which gives the effective time step for a given Fourier mode $\mathbf{k}$ as compared to the equivalent explicit Euler time stepping scheme. The Euler scheme for (1.2) is

$$
\frac{u^{n+1}-u^{n}}{\tau_{E}}=\Delta\left((\Delta+1)^{2} u^{n}-\delta u^{n}+\left(u^{n}\right)^{3}\right),
$$

where $\tau_{E}$ is the Euler time step. The update for the $\mathbf{k}^{\text {th }}$ Fourier mode is seen to be

$$
\frac{\widehat{u^{n+1}}[\mathbf{k}]-\widehat{u^{n}}[\mathbf{k}]}{\tau_{E}}=-|\mathbf{k}|^{2}\left(\left(-\left|\mathbf{k}^{2}\right|+1\right)^{2} \widehat{u^{n}}[\mathbf{k}]-\delta \widehat{u^{n}}[\mathbf{k}]+\widehat{\left(u^{n}\right)^{3}}[\mathbf{k}]\right)
$$

where the hat denotes the Fourier coefficient, $\hat{u}[\mathbf{k}] \equiv \int_{\Omega} u(\mathbf{x}) \mathrm{e}^{-i \mathbf{k} \cdot \mathbf{x}} \mathrm{d} \mathbf{x}$. The unconditionally stable algorithm (2.4) can be expressed in an analogous form,

$$
\frac{\widehat{u^{n+1}}[\mathbf{k}]-\widehat{u^{n}}[\mathbf{k}]}{\tau_{\text {eff }}[\mathbf{k}]}=-|\mathbf{k}|^{2}\left(\left(-|\mathbf{k}|^{2}+1\right)^{2} \widehat{u^{n}}[\mathbf{k}]-\delta \widehat{u^{n}}[\mathbf{k}]+\widehat{\left(u^{n}\right)^{3}}[\mathbf{k}]\right),
$$

where the effective time step for the $\mathbf{k}^{\text {th }}$ Fourier mode is given by

$$
\tau_{\text {eff }}[\mathbf{k}]=\frac{\tau}{1+\tau|\mathbf{k}|^{2}\left(\left(1-|\mathbf{k}|^{2}\right)^{2}+C \xi+\delta\right)}
$$


with $\xi=1$ if $L \equiv 1$ and $\xi=|\mathbf{k}|^{2}$ if $L \equiv \nabla$. Assuming $\delta \ll C$, for the dominant modes $\mathbf{k}$, with $|\mathbf{k}| \approx 1$, one obtains

$$
\tau_{\text {eff }}[\mathbf{k}] \approx \frac{\tau}{1+C \tau},
$$

suggesting that the maximum effective time step for the dominant modes is $\tau_{\mathrm{eff}}[\mathbf{k}]=C^{-1}$ as $\tau \rightarrow \infty$, as was observed for all frequencies in the Swift-Hohenberg evolution.

To provide guidelines for the choice of $C$ which have proved reliable in a variety of numerical simulations, we consider (2.4) in the limit that $\tau \rightarrow \infty$, i.e. the left-hand side of the equation approaches 0 . What remains may be rearranged as

$$
\Delta\left(L^{*} L-\frac{\delta}{C}+\frac{(\Delta+1)^{2}}{C}\right) u^{n+1}=\Delta\left(L^{*} L u^{n}-\frac{1}{C}\left(u^{n}\right)^{3}\right) .
$$

As periodic boundary conditions preclude linear terms, the above is solvable up to $\bar{u}^{n+1}$. However, the phase field crystal model in question fixes $\bar{u}$ throughout the evolution. In Fourier space, this gives the nearly-pointwise (up to the nonlinear term $\left(u^{n}\right)^{3}$ ) updates

$$
\widehat{u^{n+1}}[\mathbf{k}]= \begin{cases}\widehat{u^{n}}[\mathbf{0}] & \mathbf{k}=\mathbf{0}, \\ \frac{\widehat{u^{n}}[\mathbf{k}]-\frac{1}{C \xi} \widehat{\left(u^{n}\right)^{3}}[\mathbf{k}]}{1-\frac{\delta}{C \xi}+\frac{\left(-|\mathbf{k}|^{2}+1\right)^{2}}{C \xi}} & \text { otherwise. }\end{cases}
$$

The denominator indicates that high-frequency modes - those with $|\mathbf{k}| \gg \sqrt[4]{C}$ for $L \equiv 1$ and $|\mathbf{k}| \gg \sqrt{C}$ for $L \equiv \nabla-$ are immediately damped out.

First, we consider the case $L \equiv 1$. For $\mathbf{k} \approx 1$, we test the ansatz $u^{n}(x)=A \sin (\mathbf{k} \cdot \mathbf{x})$, with $\left(u^{n}(x)\right)^{3}=$ $\frac{3}{4} A^{3} \sin (\mathbf{k} \cdot \mathbf{x})-\frac{1}{4} A^{3} \sin (3 \mathbf{k} \cdot \mathbf{x})$, which gives

$$
\widehat{u^{n+1}}[\mathbf{k}] \approx A \frac{1-\frac{3 A^{2}}{4 C}}{1-\frac{\delta}{C}} .
$$

To prevent this coefficient from alternating signs at each discrete time step, we propose to choose $C \geq \frac{3}{4} A^{2}$ for $A$ the maximum amplitude among all Fourier modes $\mathbf{k} \approx 1$ of the initial data $u^{0}$. Furthermore, the denominator indicates that $C$ should be chosen such that $C>\delta$ to prevent blowup of the dominant $|\mathbf{k}| \approx 1$ modes. Finally, for the reasons suggested in Theorem 3.1, we also recommend $C \geq 3\left\|u^{0}\right\|_{\mathrm{L}^{\infty}(\Omega)}^{2}$. Together, these conditions suggest that $C$ could be chosen as, for example,

$$
C=\max \left(2 \delta,\left.\max _{|\mathbf{k}| \approx 1} \frac{3}{4} \widehat{\mid u^{0}}[\mathbf{k}]\right|^{2}, 3\left\|u^{0}\right\|_{\mathrm{L}^{\infty}(\Omega)}^{2}\right) .
$$

Our heuristic analysis suggests that it is necessary that $C$ be chosen with this scaling with respect to the initial data and parameters; furthermore, numerical experiments suggest that this choice of $C$ is also sufficient for a wide range of numerical experiments.

In the case that $L \equiv \nabla$, we observe that the update (3.8) is equivalent to the case of $L \equiv 1$ with a frequency-dependent "constant" $C|\mathbf{k}|^{2}$. Supposing that $u$ is smooth, the smallest non-zero frequency supported in one dimension by the periodic boundary conditions is $k=(2 \pi) / \ell$, where $\ell$ denotes the domain length. Thus, if (3.10) is sufficient to guarantee unconditional stability for $L=1$, choosing

$$
C=\left(\frac{\ell}{2 \pi}\right)^{2} \max \left(2 \delta,\left.\max _{|\mathbf{k}| \approx 1} \frac{3}{4} \widehat{\mid u^{0}}[\mathbf{k}]\right|^{2}, 3\left\|u^{0}\right\|_{\mathrm{L}^{\infty}(\Omega)}\right)
$$

in the case that $L \equiv \nabla$ must also be sufficient. Numerical experiments again suggest that this choice for $C$ is approximately necessary. This condition is obviously much more severe than the condition of (3.10), and for this reason we always take $L \equiv 1$ in the numerical simulations presented in Section 5 . 
We conclude this discussion with a few observations. First, while the heuristic calculations are essentially performed in one dimension, our numerical experiments suggest that $C$ may be chosen in a dimension-independent way. Next, we consider the particular case of normally distributed initial data with mean $\mu$, and standard deviation $\sigma$. In this case, $\left\|u^{0}\right\|_{L^{\infty}(\Omega)} \approx|\mu|+5 \sigma$, however, numerical experiments suggest that the optimal $C \ll 3(|\mu|+5 \sigma)^{2}$. We suggest that the guidelines presented are based on "worst-case" estimates. In the numerical experiments of Section 5 with this sort of initial data, we choose $C$ much smaller than the guidelines here suggest and numerically find energy stability. Finally, we remind the reader that these guidelines are based on heuristic calculations and empirical observations, and we do not guarantee these to be sufficient conditions for energy stability of the schemes proposed in (2.4) for all possible initial conditions.

\section{Spatial Discretization}

Until this point, the discussion has been focused on time discrete but spatially continuous functions $u^{n}(\mathbf{x})$. Here we first describe the spatial discretization we employ to obtain a fully discrete description of the proposed algorithm. Next, we present spatially discrete versions of Theorems 2.1 and 2.3 . These new theorems verify that the spatial discretization does not interfere with the existence of a $C>0$ guaranteeing the energy stablity of the proposed algorithm.

\subsection{Finite difference approximation}

For simplicity of exposition, we work on a uniform grid discretizing $\Omega=[0, \ell)^{d}$ in $d$ dimensions with $m$ grid points in each direction (with a total of $M=m^{d}$ grid points) and grid spacing $h$, so that $\ell=m h$. It is trivial to allow $\ell, m$, and $h$ to depend on dimension. We apply periodic boundary conditions and denote the discrete approximation of $u(t, \mathbf{x})$ at $t=n \tau$ and $\mathbf{x}=\mathbf{j} h$ by $U_{\mathbf{j}}^{n}$ for $n \in \mathbb{N}, \mathbf{j} \in\{0, \ldots, m-1\}^{d}$.

We discretize the spatial Laplacian using the standard second difference to define

$$
\Delta_{h} U_{\mathbf{j}}=\sum_{i=1}^{d} \frac{U_{\mathbf{j}+\mathbf{e}_{i}}-2 U_{\mathbf{j}}+U_{\mathbf{j}-\mathbf{e}_{i}}}{h^{2}},
$$

where $\mathbf{e}_{i}$ is the $i^{\text {th }}$ standard unit basis vector, and $\Delta_{h} U_{\mathbf{j}}=\Delta u(\mathbf{j} h)+O\left(h^{2}\right)$ for $u \in \mathrm{C}^{4}(\Omega)$. Here, the index arithmetic is to be interpreted in $\mathbb{Z} / m \mathbb{Z}$, taking account of the periodic boundary conditions. The temporally and spatially discretized Swift-Hohenberg and phase field crystal evolution are now given by (2.3) and (2.4) with $U$ replacing $u$ and (4.1) in place of $\Delta u$.

It is entirely straightforward to solve the discrete linear systems defined in this way. The matrix to be inverted is sparse and constant-coefficient, and so needs only be computed once. Its inverse could thus be precomputed, however, sparsity is lost following inversion so that the inverse contains $M \times M$ non-zero entries. The matrix-vector product then requires $M^{2}$ operations to compute at each time step, in addition to the $M^{2}$ memory requirement. These requirements quickly become impracticable as $m$ grows, particularly in the physically-relevant case where $d=3$.

As our preferred alternative, we remind the reader of the discrete Fourier transform (DFT), defined by

$$
\widehat{U}[\mathbf{k}]=\sum_{\mathbf{j}} U_{\mathbf{j}} \exp (-2 \pi i \mathbf{k} \cdot \mathbf{j} / m)
$$

for $\mathbf{k} \in\{0, \ldots, m-1\}^{d}$, and observe that

$$
\widehat{\Delta_{h}^{p} U}[\mathbf{k}]=\left(\frac{2}{h^{2}} \sum_{i=1}^{d}\left(\cos \left(2 \pi k_{i} / m\right)-1\right)\right)^{p} \widehat{U}[\mathbf{k}]=F[\mathbf{k}]^{p} \widehat{U}[\mathbf{k}]
$$


for $p=1,2, \ldots$, and the discretization (4.1). Making use of the DFT, we obtain the following expression for the update of the $\mathbf{k}^{\text {th }}$ Fourier coefficient by the discretized version of (2.3) and (2.4),

$$
\begin{aligned}
& \widehat{U^{n+1}}[\mathbf{k}]=\frac{\widehat{U^{n}}[\mathbf{k}]+\tau(-F[\mathbf{k}])^{\iota}\left(C(-F[\mathbf{k}])^{\sigma} \widehat{U^{n}}[\mathbf{k}]-\widehat{\left(U^{n}\right)^{3}}[\mathbf{k}]\right)}{1+\tau(-F[\mathbf{k}])^{\iota}\left((F[\mathbf{k}]+1)^{2}+C(-F[\mathbf{k}])^{\sigma}-\delta\right)} \\
& \text { with } \iota=\left\{\begin{array}{ll}
0, & \text { if discretizing }(2.3), \\
1, & \text { if discretizing }(2.4),
\end{array} \quad \sigma= \begin{cases}0, & \text { if } L \equiv 1, \\
1, & \text { if } L \equiv \nabla .\end{cases} \right.
\end{aligned}
$$

This update is pointwise in Fourier space. The computation of the Fourier and inverse Fourier transforms is the most expensive part of the computation but can be done via the Fast Fourier Transform in $O(M \log M)$ operations.

\subsection{Transfer of stability and error analysis}

Let us denote a spatially discretized function by $U=\left(U_{\mathbf{j}}\right)_{\mathbf{j}}$ and the $M$-dimensional space of such functions by $\mathcal{V}_{h}$. For $U, V \in \mathcal{V}_{h}$ we define the inner product

$$
(U, V)_{h}=h^{d} \sum_{\mathbf{j}} U_{\mathbf{j}} \cdot V_{\mathbf{j}}
$$

and the discrete $\mathrm{L}^{p}-$ norm

$$
\|U\|_{\mathrm{L}_{h}^{p}}^{p}=\left(|U|^{p}, \mathbb{1}\right)_{h}, \quad\|U\|_{L_{h}^{\infty}}=\max _{\mathbf{j}}\left|U_{\mathbf{j}}\right|
$$

where $\mathbb{1}$ denotes the discretized function which is constantly one. Let us introduce the discrete gradient

$$
\nabla_{h} U_{\mathbf{j}}=\frac{1}{h}\left(U_{\mathbf{j}}-U_{\mathbf{j}-\mathbf{e}_{1}}, \ldots, U_{\mathbf{j}}-U_{\mathbf{j}-\mathbf{e}_{d}}\right)^{T}
$$

where again the index -1 shall be interpreted as $m-1$. One can easily verify the integration by parts formula

$$
\left(\nabla_{h} U, \nabla_{h} V\right)_{h}=-\left(U, \Delta_{h} V\right)_{h}
$$

Finally, there are constants $\tilde{\nu}, \tilde{\gamma}>0$ with

$$
\begin{gathered}
\|U\|_{\mathrm{L}_{h}^{2}}^{2}+\left\|\nabla_{h} U\right\|_{\mathrm{L}_{h}^{2}}^{2}+\left\|\Delta_{h} U\right\|_{\mathrm{L}_{h}^{2}}^{2} \geq \tilde{\gamma}\|U\|_{\mathrm{L}_{h}^{\infty}}^{2}, \\
\left\|\nabla_{h} U\right\|_{\mathrm{L}_{h}^{2}}^{2} \geq \tilde{\nu}\left\|U-(U, \mathbb{1})_{h} / \mid \Omega\right\|_{\mathrm{L}_{h}^{2}}^{2},
\end{gathered}
$$

a discrete Sobolev and Poincaré inequality, the proofs of which can be performed analogously to the proofs of the corresponding results in [12].

The spatially discrete version of the free energy is now given as

$$
\mathcal{E}_{h}[U]=\frac{1}{2}\left\|\Delta_{h} U+U\right\|_{\mathrm{L}_{h}^{2}}^{2}-\frac{\delta}{2}\|U\|_{\mathrm{L}_{h}^{2}}^{2}+\frac{1}{4}\|U\|_{\mathrm{L}_{h}^{4}}^{4} .
$$

Replacing the differential operators, inner products, and norms in the proof of Theorem 2.1 by their discrete counterparts, we arrive at the following stability result.

Theorem 4.1 (Stability of (4.4)). Assume $\delta<1$. For any $U^{0}: \Omega \rightarrow \mathbb{R}$ with finite energy $\mathcal{E}_{h}$ there exists a $C>0$ such that the fully discrete scheme (4.4) is stable for any $\tau>0$ in the sense

$$
\begin{aligned}
& \mathcal{E}_{h}\left[U^{n+1}\right] \leq \mathcal{E}_{h}\left[U^{n}\right] \quad \forall n \in \mathbb{N}, \\
& \exists U>0:\left\|U^{n}\right\|_{\mathrm{L}_{h}^{\infty}(\Omega)} \leq U \forall n \in \mathbb{N} .
\end{aligned}
$$


Likewise, the proof of the error estimate Theorem 2.3 can be transferred one-to-one, noting that the truncation error for temporal and spatial discretization can be bounded by

$$
\begin{array}{r}
\left|\rho_{\mathbf{j}}^{n+1}\right| \leq \tau \frac{1}{2}\|u\|_{\mathrm{C}^{2}\left([0, T], \mathrm{C}^{0}(\Omega)\right)}+h^{2}\|u\|_{\mathrm{C}^{0}\left([0, T], \mathrm{C}^{8}(\Omega)\right)} \\
+\tau h^{2} C\|u\|_{\mathrm{C}^{1}\left([0, T], \mathrm{C}^{a}(\Omega)\right)}+\tau h^{2}\|u\|_{\mathrm{C}^{1}\left([0, T], \mathrm{C}^{4}(\Omega)\right)}^{3} \leq \tilde{K}\left(\tau+h^{2}\right)
\end{array}
$$

for some $\tilde{K} 0$ and with $a=4$ for $L \equiv 1$ and $a=6$ for $L \equiv \nabla$.

Theorem 4.2 (Error estimate of (4.4)). Suppose the Swift-Hohenberg or phase field crystal equation is solved by a smooth spatially periodic function $u:[0, T] \times \Omega \rightarrow \mathbb{R}$ for some $T \in(0, \infty)$, and denote the solution to (4.4) by $U^{n}, n=0,1, \ldots$, where $U^{0}=(u(0, \mathbf{j} h))_{\mathbf{j}}$ and $C$ is chosen according to the previous theorem. Then, there exists a constant $K>0$ independent of $\tau$ and $h$ such that (for $\tau, h$ small enough)

$$
\left\|(u(n \tau, \mathbf{j} h))_{\mathbf{j}}-U^{n}\right\|_{L_{h}^{2}} \leq K\left(\tau+h^{2}\right)
$$

for all $n$ with $n \tau \leq T$.

\section{Numerical VALIDATION}

The primary goal of this section is to present some numerical experiments validating this algorithm. We present several simple simulations in one, two, and three spatial dimensions. We demonstrate the numerical convergence of the algorithm as $\tau \rightarrow 0$ and also compare its evolution for large $\tau$ to an alternative linear scheme from the literature. We then show the ability of the algorithm to preserve a coexistence solution in one dimension, and perform fairly large, sufficiently resolved two- and three-dimensional simulations. Next, we present a series of small simulations in three dimensions demonstrating various types of microstructure that can be obtained in three dimensions even with fairly low spatial resolution.

\subsection{Numerical convergence test}

Though this work is primarily concerned with demonstrating the unconditional stability of the proposed algorithm, it is also important to understand the convergence properties of the algorithm as $\tau \rightarrow 0$. To that end, we performed a numerical convergence test on the two-dimensional developing periodic structure displayed in Figure 1a. The parameters of the simulation are $\ell=24 \pi, m=500, C=1, \delta=1 / 2, \bar{u}=1 / 4$, and the total simulation time is 1 . Twelve tests were run, with $\tau=2^{1-p}$ for the $p^{\text {th }}$ test. The results, shown in Table 1 , are indicative of linear convergence to some unknown exact solution. Figure 1(b-c) demonstrates that the relative error in energy dissipation and the discrete $L_{h}^{2}$ error with respect to the most refined computed solution decrease approximately linearly for the intermediary tests. It is expected that if the exact solution were known and compared to, this linear convergence would continue through the more refined tests.

\subsection{Comparison to an alternative linear scheme}

In [3], Cheng and Warren propose a linear scheme for the phase field crystal evolution, given by

$$
\begin{aligned}
\frac{u^{n+1}-u^{n}}{\tau}= & \Delta\left[(1-\delta)\left(a_{1} u^{n}+\left(a_{1}-1\right) u^{n+1}\right)\right. \\
& \left.+2\left(a_{2} \Delta u^{n}+\left(a_{2}-1\right) \Delta u^{n+1}\right)+\left(a_{3} \Delta^{2} u^{n}+\left(a_{3}-1\right) \Delta^{2} u^{n+1}\right)+\left(u^{n}\right)^{3}\right],
\end{aligned}
$$

where from a linear stability analysis they derive the conditions

$$
a_{1}<\frac{1}{2}-\frac{3 \bar{u}^{2}}{2(1-\delta)}, \quad a_{2} \geq \frac{1}{2}, \quad a_{3} \leq \frac{1}{2}
$$




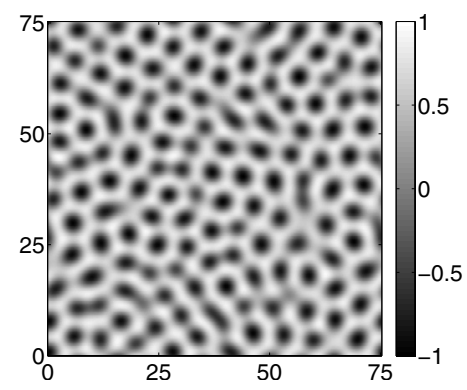

(a)

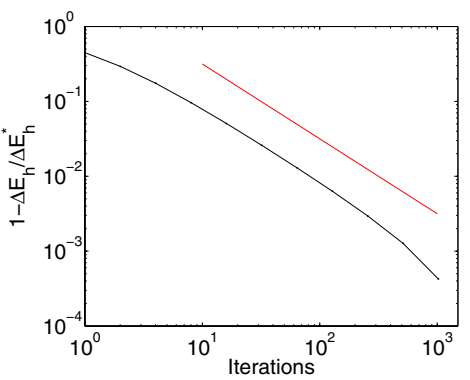

(b)

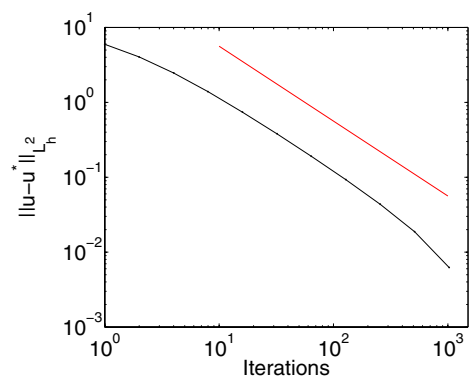

(c)

Figure 1. (a) The initial microstructure for the numerical convergence test. (b) Relative error in energy dissipation and (c) $L_{h}^{2}$ error, as compared to the most refined solution. The line with slope -1 is provided as a guide for the eye, suggestive of linear convergence to some (unknown) exact solution.

TABLE 1. Each test ran $n$ iterations at time step $\tau$ for total evolution time 1 . The total discrete energy dissipated throughout the evolution is denoted $\Delta \mathcal{E}_{h}$. Reference values $\Delta \mathcal{E}_{h}^{*}$ and $U^{*}$ refer to the most refined simulation. By approximately $n=8$, convergence in $1-\Delta \mathcal{E}_{h} / \Delta \mathcal{E}_{h}^{*}$ and $\left\|U^{n}-U^{*}\right\|_{L_{h}^{2}}$ appear approximately linear (compare Fig. 1).

\begin{tabular}{lcccc}
\hline$n$ & $\tau$ & $\Delta \mathcal{E}_{h}$ & $1-\Delta \mathcal{E}_{h} / \Delta \mathcal{E}_{h}^{*}$ & $\left\|U^{n}-U^{*}\right\|_{L_{h}^{2}}$ \\
\hline 1 & 1 & 1.8755 & $4.472 \times 10^{-1}$ & $1.356 \times 10^{-1}$ \\
2 & $1 / 2$ & 2.3964 & $2.936 \times 10^{-1}$ & $9.199 \times 10^{-2}$ \\
4 & $1 / 4$ & 2.8001 & $1.746 \times 10^{-1}$ & $5.620 \times 10^{-2}$ \\
8 & $1 / 8$ & 3.0651 & $9.646 \times 10^{-2}$ & $3.163 \times 10^{-2}$ \\
16 & $1 / 16$ & 3.2201 & $5.079 \times 10^{-2}$ & $1.684 \times 10^{-2}$ \\
32 & $1 / 32$ & 3.3044 & $2.594 \times 10^{-2}$ & $8.652 \times 10^{-3}$ \\
64 & $1 / 64$ & 3.3484 & $1.296 \times 10^{-2}$ & $4.336 \times 10^{-3}$ \\
128 & $1 / 128$ & 3.3709 & $6.317 \times 10^{-3}$ & $2.117 \times 10^{-3}$ \\
256 & $1 / 256$ & 3.3823 & $2.959 \times 10^{-3}$ & $9.927 \times 10^{-4}$ \\
512 & $1 / 512$ & 3.3881 & $1.271 \times 10^{-3}$ & $4.264 \times 10^{-4}$ \\
1024 & $1 / 1024$ & 3.3909 & $4.239 \times 10^{-4}$ & $1.423 \times 10^{-4}$ \\
2048 & $1 / 2048$ & 3.3924 & 0 & 0 \\
\hline
\end{tabular}

for the weights $a_{1}, a_{2}, a_{3}$. The underlying motivation is to evolve the phase field crystal model in steps as large as possible, where each time step only requires the solution of a linear system of equations. From this efficiency perspective it makes sense to compare (5.1) to our newly proposed scheme with regard to the number of effective time steps needed to evolve to a similar state. Figure 2 shows solutions $u^{n}$ for both schemes after different numbers $n$ of time steps. The top row depicts the evolution of our scheme, while the bottom two rows are simulated via the algorithm from [3], using two different sets of numerical parameters: The first set has been chosen experimentally to maximize the effective crystal evolution per time step (Fig. 2, middle row), whereas the second set appears to achieve the fastest energy decrease (bottom row).

Remarkably, despite the large time step size and the inevitably associated inaccuracy, all three simulations evolve through very similar states. It cannot be expected that these states occur after the same number of time steps, though, as the schemes might have very different effective time steps for large $\tau$ (compare the discussion in Sect. 3). Apparently, while each step of (5.1) with the first parameter set evolves the crystal almost as far as our scheme does, the energy hardly decreases due to high frequency modes which are only slowly damped. The second parameter set immediately smoothes out these high frequencies and thus leads to a low energy level 


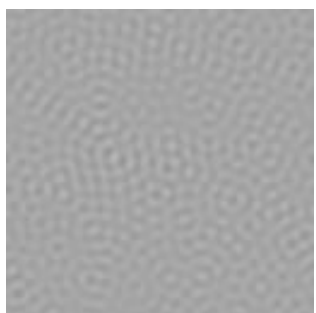

$n=5, \mathcal{E}=39.23$

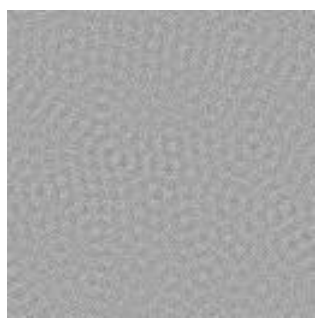

$n=7, \mathcal{E}=58.42$

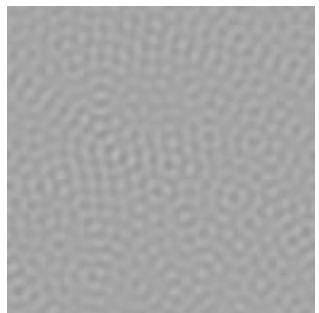

$n=265, \mathcal{E}=39.23$

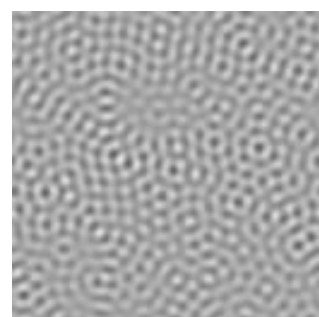

$n=10, \mathcal{E}=39.17$

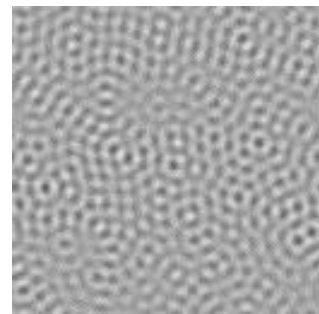

$n=14, \mathcal{E}=56.21$

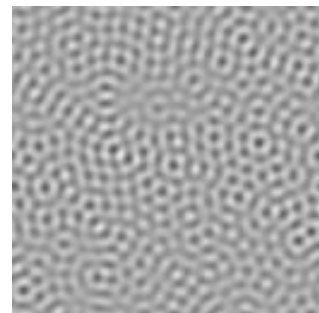

$n=530, \mathcal{E}=39.16$

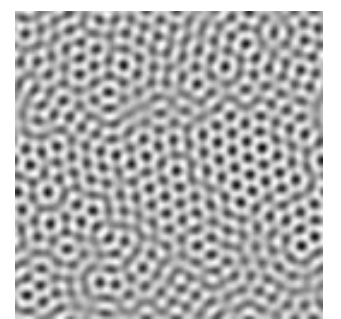

$n=15, \mathcal{E}=38.94$

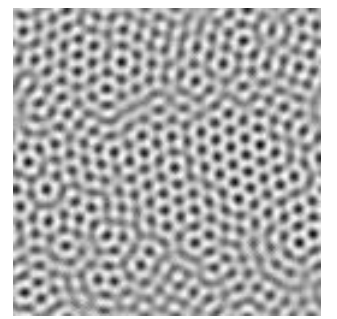

$n=21, \mathcal{E}=54.37$

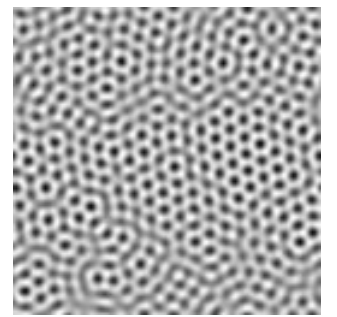

$n=795, \mathcal{E}=38.90$

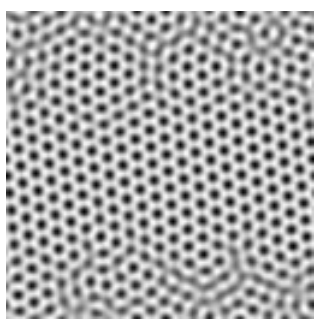

$n=20, \mathcal{E}=38.71$

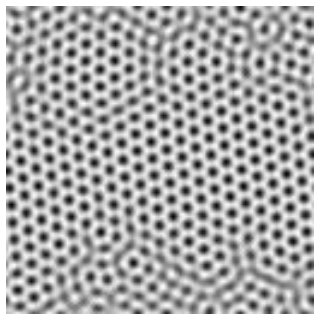

$n=28, \mathcal{E}=53.05$

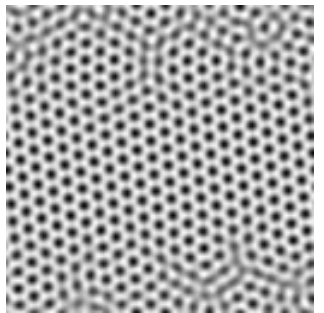

$n=1060, \mathcal{E}=38.69$

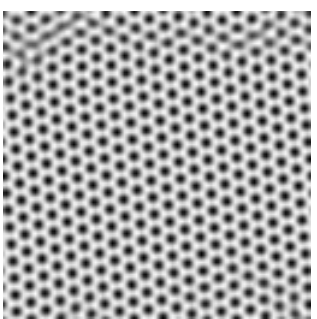

$n=25, \mathcal{E}=38.54$

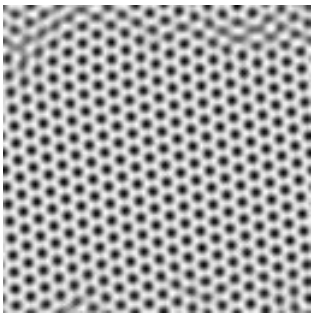

$n=35, \mathcal{E}=52.06$

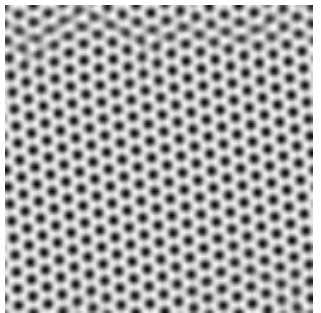

$n=1325, \mathcal{E}=38.53$

Figure 2. Comparison of our new scheme (2.4) (top row: $L \equiv 1, C=.05, \tau=10^{5}$ ) with the linear scheme from [3] (middle row: $a_{1}=0.45, a_{2}=0.5, a_{3}=0.5, \tau=10^{5}$; bottom row: $\left.a_{1}=0.45, a_{2}=1, a_{3}=0, \tau=10^{5}\right)$. We show density snapshots after different numbers $n$ of time steps, starting from the same initial condition. The constants $a_{1}, a_{2}, a_{3}$ for the middle and bottom row have been chosen optimally to give the fastest evolution and the fastest energy decrease, respectively. Parameters were chosen as in [3]: $\delta=0.025, \bar{u}=0.07, \ell=128, m=128$.

as quickly as our method, however, the system is seen to evolve much more slowly. In all three cases, a further increase of the nominal time step size $\tau$ does not have any visible effect on the results.

\subsection{Coexistence in one dimension}

Elder and Grant [4] studied the approximate phase diagrams for the phase field crystal model $(\delta=-r$ in their notation) via one-mode approximations. They demonstrated that, for any $\delta>1 / 4$, there is a range of values of $\bar{u}$ for which it is energetically favorable to choose a solution that is essentially piecewise, corresponding to a liquid solution in one region and a periodic solution in the other. We numerically demonstrate the ability of our algorithm to preserve such solutions.

In this one-dimensional simulation, we take as parameters $\ell=28 \pi, m=1000, \bar{u}=1 / 2$, and $\delta=3 / 4$. We compare three different initial conditions for $u$ : a constant liquid state $\left(u_{1}=1 / 2\right)$, a one-mode approximation of a periodic state $\left(u_{2}=1 / 2+1 / 25 \sin (x)\right)$, and a coexistence state chosen so that the appropriate mean $\bar{u}$ is 


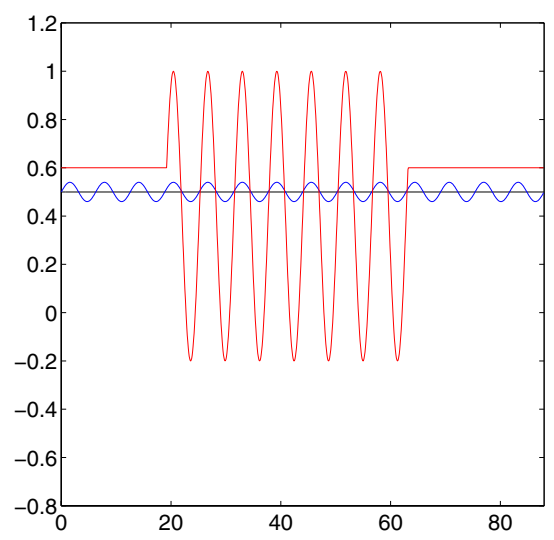

(a)

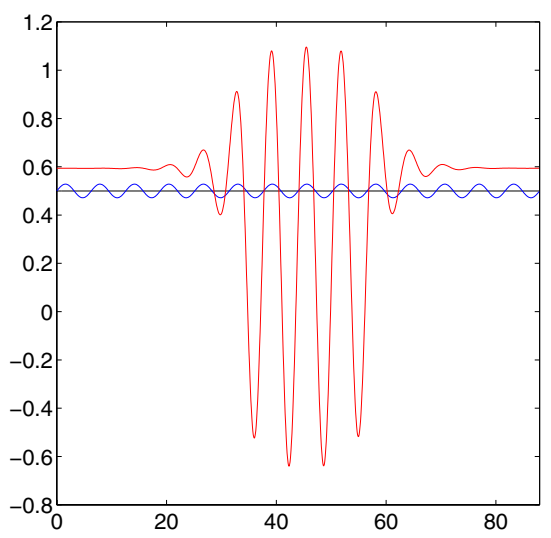

(b)

Figure 3. (a) The initial microstructures for a one-dimensional simulation of coexistence. (b) At $t=1000$, the liquid solution remains stationary while the periodic solution changes minimally. The calculated energy density for each remains $\varepsilon_{h}=0.0469$ to four decimal places. The coexistence solution has changed significantly and has a final calculated energy density $\varepsilon_{h}=0.0437$. In each plot, $U_{1}$ is the black curve, $U_{2}$ the blue curve, and $U_{3}$ the red curve.

maintained and the function is continuous:

$$
u_{3}= \begin{cases}2 / 5+3 / 5 \sin (x), & \text { if } 6 \pi+\sin ^{-1}\left(\frac{1}{3}\right) \leq x<20 \pi+\sin ^{-1}\left(\frac{1}{3}\right), \\ 3 / 5, & \text { otherwise. }\end{cases}
$$

Discrete versions of these initial conditions are superimposed in Figure 3a. The proposed algorithm is run for 1000 iterations with $\tau=1$ and $C=1$ with each initial condition, generating the resulting microstructure seen in Figure 3b. The initial and final discrete energy densities $\left(\varepsilon_{h}=\mathcal{E}_{h} / \ell\right)$ for both $U_{1}$ and $U_{2}$ are 0.0469 to four decimal places. $U_{1}$ clearly remains stationary, which is not surprising, given that the liquid is a stationary solution. The apparent amplitude of $U_{2}$ shrinks slightly (to approximately 0.03), and the details of the profile are expected to change some, though any changes from the one-mode approximation are not easily visible. It is expected that after a longer simulation time, numerical perturbations will cause the system to leave this periodic profile in favor of the coexistence state. The initial discrete energy density of $U_{3}$ is calculated as 0.0767 (and is unbounded as $h \rightarrow 0$ as $u_{3}$ is not everywhere differentiable), but the final energy density is calculated to be 0.0437 , less than the calculated energy of the liquid or periodic solutions.

\subsection{Medium-scale simulation of grain coarsening in $2 \mathrm{D}$}

The phase field crystal model is also able to describe a classic phenomenon known as grain coarsening: If the initial condition $u^{0}$ contains crystals of different orientation with interfaces in between (compare Fig. 4, bottom), then these interfaces move during the evolution in such a way that large crystals or grains increase in size on the expense of small grains, which shrink until they finally disappear. Such simulations of polycrystals typically involve a large amount of atoms and can easily be performed using the proposed scheme. A very basic Matlab implementation already yields the result shown in Figure 4 in just two hours computation time on a single processor. Due to the scheme's simplicity, a highly parallel implementation could readily be programmed on a GPU, which would increase the simulation speed even much more. 

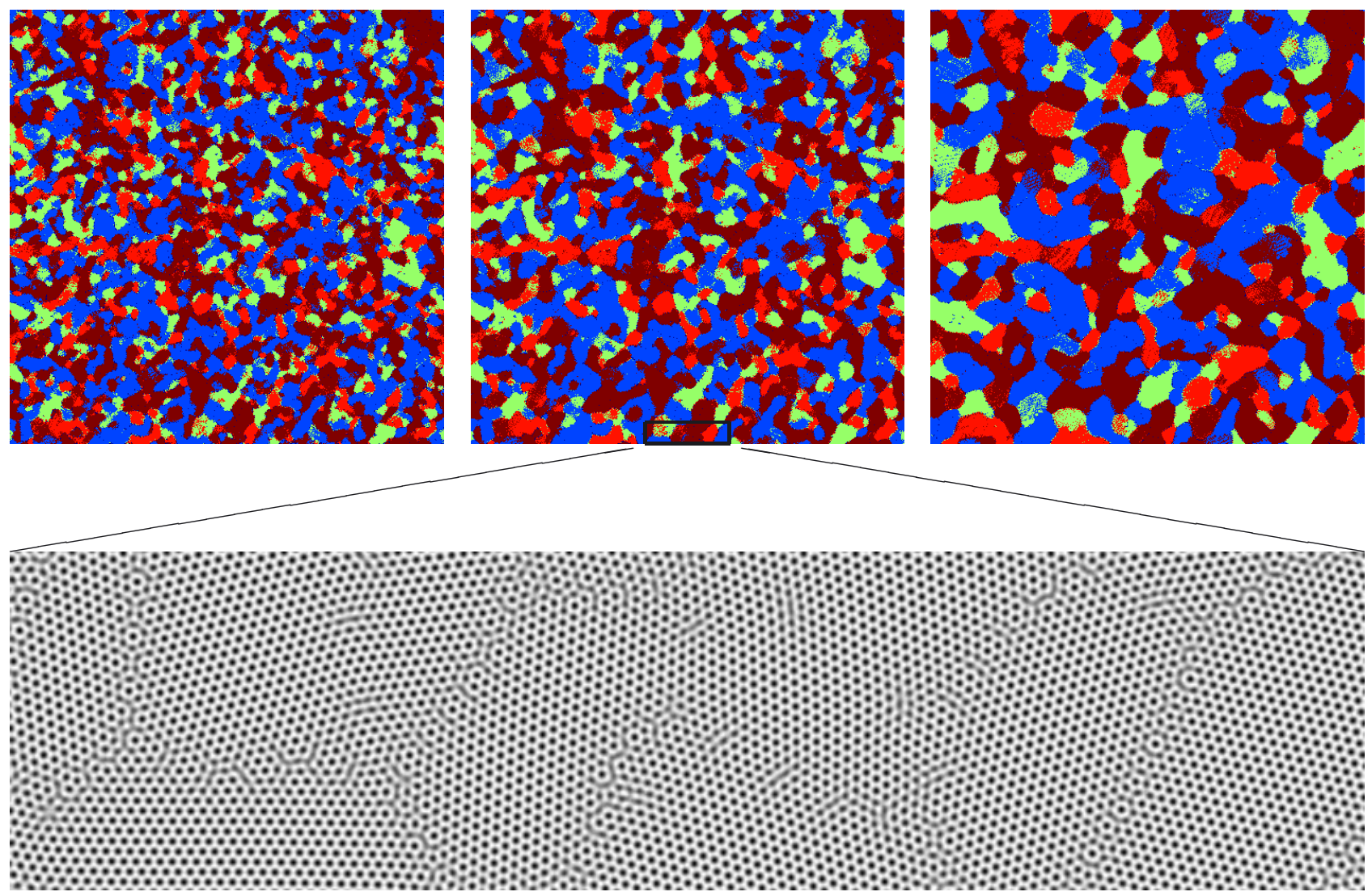

FiguRE 4. Simulation of grain coarsening (same parameters as Fig. 2 except $\ell=4097, m=$ $4097, \sim 3.7 \cdot 10^{5}$ atoms). The coloring helps to distinguish regions of different lattice orientation, the bottom image shows a zoom-in. The result is shown after 50, 100, and 500 time steps. Simulation time on single processor was $2 \mathrm{~h}$ and $12 \mathrm{~min}$.

\subsection{Well-resolved three-dimensional simulation}

To demonstrate the efficiency of our algorithm, we present a small but well-resolved three-dimensional simulation. We choose $m=100, \ell=10 \pi, \tau=1, \delta=1 / 2$, and $C=5$. The initial data is normally distributed with mean $\bar{U}=0.02$ and standard deviation 0.2. 10000 time steps are performed. Figure 5 shows the microstructural evolution through time. Initially, $U$ appears to rapidly approach the liquid solution $U \equiv \bar{U}$, but structure quickly emerges and then on a much slower time scale appears to form rolls. The energy is numerically calculated to decrease at each time step.

\subsection{Phase portrait in three dimensions}

Here we present a series of minimally-resolved calculations in three dimensions to demonstrate that this algorithm can find the various types of microstructure expected to exist in three dimensions with quite minimal computational effort. We choose $m=40$ and $\ell=8 \pi$, so that $h=\pi / 5$. We fix $\delta=1 / 4, \tau=1, C=1$, and compute until $t=10000$. We choose normally distributed initial data $\Psi^{0}$ with mean 0 and standard deviation 1 , and vary the mean $\bar{u}$ by setting $U^{0}=\Psi^{0}+\bar{U}$. We explore the choices $\bar{U}=0.1,0.2,0.3$, and 0.4 , finding four distinct types of microstructure. In analogy with the striped and hexagonal phases found in two dimensions, 


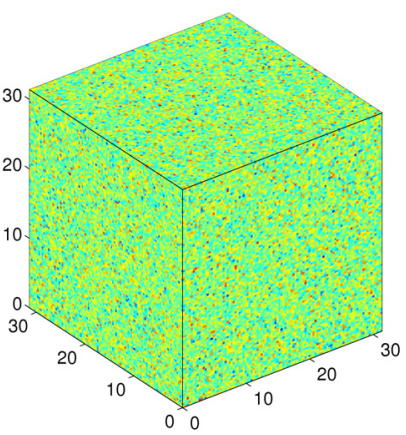

(a)

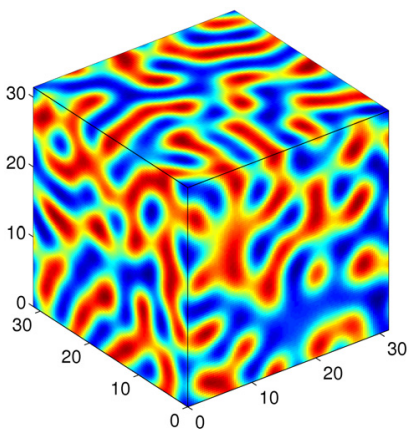

(d)

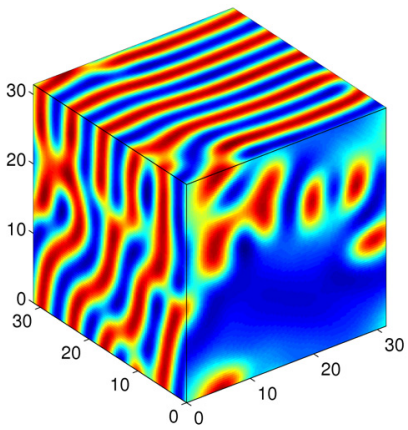

(g)

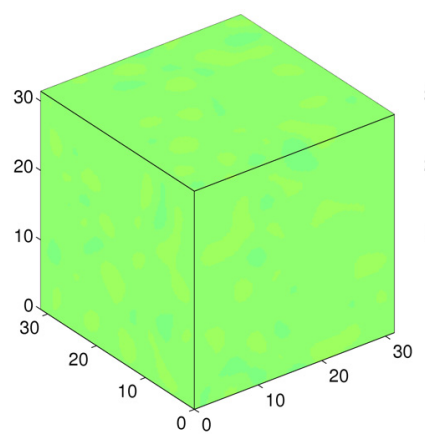

(b)

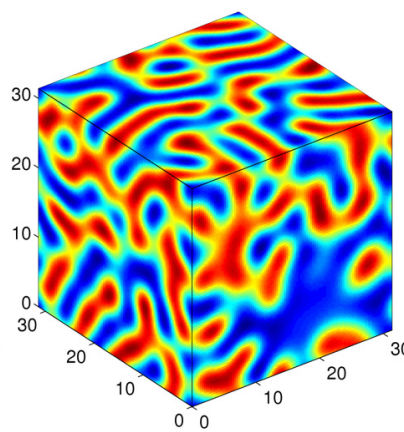

(e)

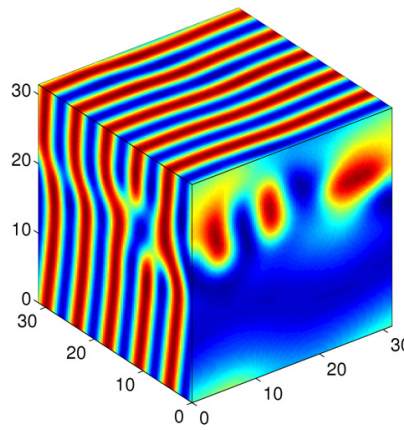

(h)

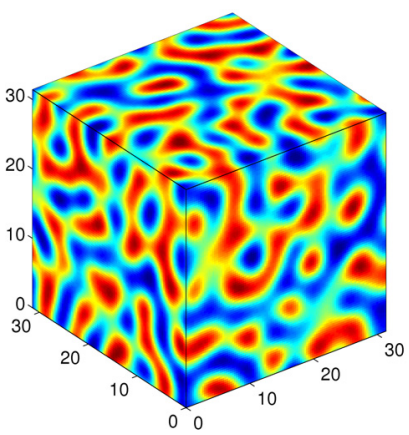

(c)

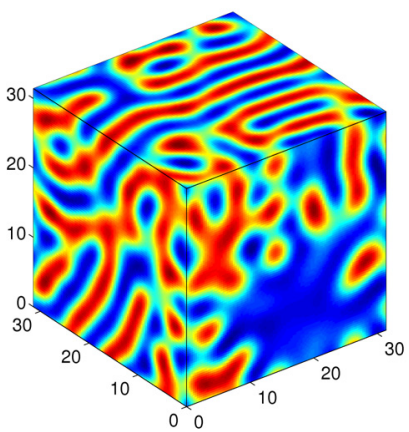

(f)

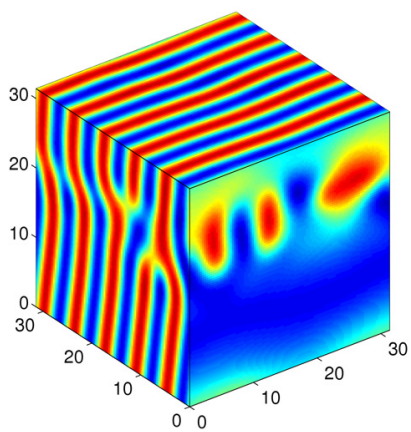

(i)

Figure 5. Microstructure from 3D simulation at (a) initial condition, (b) $t=10$, (c) $t=100$, (d) $t=1000$, (e) $t=2000$, (f) $t=4000$, (g) $t=6000$, (h) $t=8000$, (i) $t=10000$. The color scale ranges from -1 (blue) to 1 (red) in each figure. The microstructure quickly evolves from the random initial condition towards the pure liquid state around $t=10$, then on a slower time scale begins to evolve towards the "rolls" microstructure.

there are three periodic phases, with one-, two-, and three-dimensional periodicity, respectively. For large $\bar{U}$, we obtain the constant liquid microstructure. See Figure 6 for the various periodic phases. Table 2 compares the energy of the periodic solutions to the calculated energy of the liquid solution with the same parameter choices and demonstrates that the periodic solutions are, indeed, energetically favorable to the liquid solution where they appear. 


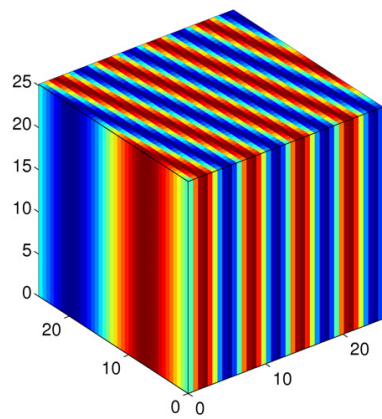

(a)

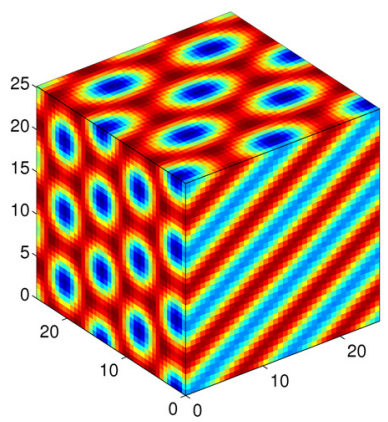

(b)

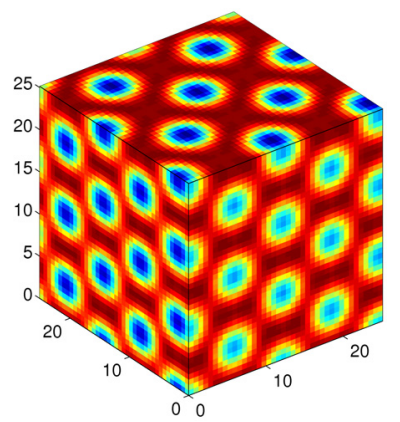

(c)

FiguRE 6 . The one-, two-, and three-dimensional periodic microstructures arising for $\bar{U}=0.1$, 0.2 , and 0.3 , respectively, and $\delta=0.25$. Even with low spatial resolution $(h=\pi / 5)$, the algorithm is able to find the various types of microstructure for different values of $\bar{U}$.

TABle 2. Comparison of the calculated discrete energy density $\varepsilon_{h}$ for the various types of microstructure displayed in Figure 6 to the discrete energy density of the liquid solution for $\delta=1 / 4$. In each of the periodic cases, the calculated energy density for the periodic solution is less than the energy associated with the liquid solution.

\begin{tabular}{cccc}
\hline $\bar{U}$ & Microstructure & $\varepsilon_{h}(U)$ & $\varepsilon_{h}(\bar{U})$ \\
\hline 0.1 & 1-periodic & $-4.30 \times 10^{-3}$ & $3.78 \times 10^{-3}$ \\
0.2 & 2-periodic & $9.87 \times 10^{-3}$ & $1.54 \times 10^{-2}$ \\
0.3 & 3-periodic & $3.44 \times 10^{-3}$ & $3.58 \times 10^{-2}$ \\
0.4 & liquid & $6.64 \times 10^{-2}$ & $6.64 \times 10^{-2}$ \\
\hline
\end{tabular}

\section{Appendix A. The limit of $\left\|A^{C}\right\|_{\mathrm{L}^{\infty}}$ AS $C \rightarrow \infty$}

For simplicity we will assume $\Omega=[0, \ell]^{d} \subset \mathbb{R}^{d}$. Let us introduce the functions

$$
\begin{aligned}
\tilde{g}^{\kappa}(\mathbf{x}) & =\kappa^{d} \sum_{\mathbf{k} \in \mathbb{N}^{d}}^{\prime \prime} \frac{\cos \left(\kappa k_{1} x_{1}\right) \cdots \cos \left(\kappa k_{d} x_{d}\right)}{1+\kappa^{4}\left(k_{1}^{2}+\cdots+k_{d}^{2}\right)^{2}}, \\
g^{\kappa}(\mathbf{x}) & = \begin{cases}\tilde{g}^{\kappa}(\mathbf{x}), & \mathbf{x} \in\left[0, \frac{\pi}{\kappa}\right]^{d}, \\
0 & \text { else },\end{cases} \\
g(\mathbf{x}) & =\int_{[0, \infty)^{d}} \frac{\cos \left(k_{1} x_{1}\right) \cdots \cos \left(k_{d} x_{d}\right)}{1+\left(k_{1}^{2}+\cdots+k_{d}^{2}\right)^{2}} \mathrm{~d} \mathbf{k},
\end{aligned}
$$

where $k_{i}$ denotes the $i$ th component of the vector $\mathbf{k}$. $\sum_{\mathbf{k} \in \mathbb{N}^{d}}^{\prime \prime}$ is an abbreviation for $\sum_{k_{1} \in \mathbb{N}}^{\prime} \ldots \sum_{k_{d} \in \mathbb{N}}^{\prime}$, where the prime indicates that the first summand $\left(k_{i}=0\right)$ is weighted with $\frac{1}{2}$. For $\mathbf{x} \in\left[0, \frac{\pi}{\kappa}\right]^{d}, g^{\kappa}(\mathbf{x})$ is the trapezium rule quadrature for $g(\mathbf{x})$ on a uniform $d$-dimensional grid with spacing $\kappa$, and one can readily show $g^{\kappa} \rightarrow_{\kappa \rightarrow 0} g$ pointwise, since the integrand decays quickly enough in $\mathbf{k}$. Let us furthermore introduce the $d$-dimensional Fourier transform $\mathcal{F}: \mathrm{L}^{2}\left(\mathbb{R}^{d}\right) \rightarrow \mathrm{L}^{2}\left(\mathbb{R}^{d}\right), \mathcal{F}[u](\mathbf{k})=\int_{\mathbb{R}^{d}} u(\mathbf{x}) \mathrm{e}^{-i 2 \pi \mathbf{k} \cdot \mathbf{x}} \mathrm{d} \mathbf{x}$, then we observe

$$
g(\mathbf{x})=\frac{1}{2^{d}} \int_{\mathbb{R}^{d}} \frac{\mathrm{e}^{-i \mathbf{k} \cdot \mathbf{x}}}{1+|\mathbf{k}|^{4}} \mathrm{~d} \mathbf{k}=\frac{1}{2^{d}} \mathcal{F}\left[\frac{1}{1+|\cdot|^{4}}\right]\left(\frac{\mathbf{x}}{2 \pi}\right) .
$$


Lemma A.1. If $g^{\kappa} \rightarrow g$ in $\mathrm{L}^{1}\left([0, \infty)^{d}\right)$ as $\kappa \rightarrow 0$, then $\left\|A^{C}\right\|_{\mathrm{L}^{\infty}} \rightarrow\left\|\mathcal{F}\left[1 /\left(1+|\cdot|^{4}\right)\right]\right\|_{\mathrm{L}^{1}\left(\mathbb{R}^{d}\right)}$ as $C \rightarrow \infty$.

Proof. Let $\hat{\mathcal{F}}: \mathrm{L}^{2}(\Omega ; \mathbb{C}) \rightarrow l^{2}\left(\mathbb{Z}^{d} ; \mathbb{C}\right), \hat{\mathcal{F}}[u](\mathbf{k})=\frac{1}{\ell^{d}} \int_{\Omega} u(\mathbf{x}) \mathrm{e}^{-i 2 \pi \mathbf{k} \cdot \frac{\mathbf{x}}{\ell}} \mathrm{d} \mathbf{x}$, denote the semi-discrete Fourier transform. For $u \in \mathrm{L}^{2}(\Omega)$ we have $\hat{\mathcal{F}}\left[A^{C} u\right](\mathbf{k})=\hat{\mathcal{F}}[u](\mathbf{k})\left[1-\frac{\delta}{C}+\frac{\left(\left|\frac{2 \pi}{\ell} \mathbf{k}\right|^{2}-1\right)^{2}}{C}\right]^{-1}$ and thus $A^{C} u=h^{C} * u$ for

$$
h^{C}(\mathbf{x})=\frac{1}{\ell^{d}} \sum_{\mathbf{k} \in \mathbb{Z}^{d}} \frac{\mathrm{e}^{i 2 \pi \mathbf{k} \cdot \frac{\mathbf{x}}{\ell}}}{1-\frac{\delta}{C}+\frac{\left(\left|\frac{2 \pi}{\ell} \mathbf{k}\right|^{2}-1\right)^{2}}{C}}=\left(\frac{2}{\ell}\right)^{d} \sum_{\mathbf{k} \in \mathbb{N}^{d}}^{\prime \prime} \frac{\cos \left(\frac{2 \pi}{\ell} k_{1} x_{1}\right) \cdots \cos \left(\frac{2 \pi}{\ell} k_{d} x_{d}\right)}{1-\frac{\delta}{C}+\frac{\left(\left(\frac{2 \pi}{\ell}\right)^{2}\left(k_{1}^{2}+\cdots+k_{d}^{2}\right)-1\right)^{2}}{C}} .
$$

Due to the high decay rate of its Fourier coefficients $h^{C}$ is continuous, and furthermore $\left\|A^{C}\right\|_{\mathrm{L}^{\infty}}=\left\|h^{C}\right\|_{\mathrm{L}^{1}(\Omega)}$. We split up $h^{C}$ according to $h^{C}=h_{1}^{C}+h_{2}^{C}$ with $h_{1}^{C}(\mathbf{x})=(\sqrt[4]{C} / \pi)^{d} \tilde{g}^{(2 \pi) /(\ell \sqrt[4]{C})}(\sqrt[4]{C} \mathbf{x})$. It is straightforward to show that the $\mathrm{L}^{2}(\Omega)$-norm (and thus also the $\mathrm{L}^{1}(\Omega)$-norm) of the remainder $h_{2}^{C}$ converges to zero as $C \rightarrow \infty$ so that $\lim _{C \rightarrow \infty}\left\|A^{C}\right\|_{\mathrm{L}^{\infty}}=\lim _{C \rightarrow \infty}\left\|h_{1}^{C}\right\|_{\mathrm{L}^{1}(\Omega)}$. Finally, abbreviating $\kappa=\frac{2 \pi}{\ell \sqrt[4]{C}}$,

$$
\begin{aligned}
\left\|h_{1}^{C}\right\|_{\mathrm{L}^{1}(\Omega)}=\frac{1}{\pi^{d}}\left\|\tilde{g}^{\kappa}\right\|_{\mathrm{L}^{1}\left(\left[0, \frac{2 \pi}{\kappa}\right]^{d}\right)}=\left(\frac{2}{\pi}\right)^{d}\left\|g^{\kappa}\right\|_{\mathrm{L}^{1}\left([0, \infty)^{d}\right)} \underset{\substack{C \rightarrow \infty \\
\kappa \rightarrow 0}}{\longrightarrow}\left(\frac{2}{\pi}\right)^{d}\|g\|_{\mathrm{L}^{1}\left([0, \infty)^{d}\right)} \\
\quad=2^{d}\left\|\mathcal{F}\left[1 /\left(1+|\cdot|^{4}\right)\right]\right\|_{\mathrm{L}^{1}\left([0, \infty)^{d}\right)}=\left\|\mathcal{F}\left[1 /\left(1+|\cdot|^{4}\right)\right]\right\|_{\mathrm{L}^{1}\left(\mathbb{R}^{d}\right)},
\end{aligned}
$$

where we have applied a change of variables in the first equality on either side of the limit and where we have exploited the evenness and $\frac{2 \pi}{\kappa}$-periodicity of $\tilde{g}^{\kappa}$.

Due to the pointwise convergence $g^{\kappa} \rightarrow g$, by Lebesgue's theorem it would be sufficient to majorize all $g^{\kappa}$ by an $\mathrm{L}^{1}(\Omega)$ function in order to verify the conditions of the previous lemma. However, such a function is not easily found. In $1 \mathrm{D}$, one can find a direct proof of the $\mathrm{L}^{1}$-convergence $g^{\kappa} \rightarrow g$ (see below). In higher dimensions, similar methods can probably be applied (using higher-dimensional Euler-Maclaurin formulae [10]), however, the resulting equations will become highly complicated.

Lemma A.2. In $1 D(d=1), g^{\kappa} \rightarrow g$ in $\mathrm{L}^{1}([0, \infty))$ as $\kappa \rightarrow 0$.

Proof. Define $f^{x, \kappa}(k)=\kappa \frac{\cos (k \kappa x)}{1+(k \kappa)^{4}}$. For $x \in\left[0, \frac{\pi}{\kappa}\right]$, the classical second order Euler-Maclaurin formula yields

$$
\left|g^{\kappa}(x)-g(x)\right|=\left|\int_{0}^{\infty} \frac{B_{2}(k-\lfloor k\rfloor)}{2}\left(f^{x, \kappa}\right)^{\prime \prime}(k) \mathrm{d} k\right|,
$$

where $B_{2}$ is the second Bernoulli polynomial. It is known that $B_{2}(k-\lfloor k\rfloor) / 2=\sum_{n=1}^{\infty} \frac{\cos (2 \pi n k)}{(2 \pi n)^{2}}$. Using this fact, after some algebra we arrive at

$$
\begin{aligned}
\left|g^{\kappa}(x)-g(x)\right|= & \mid \sum_{n=1}^{\infty} \frac{1}{(2 \pi n)^{2}} \int_{0}^{\infty}\left[\left(\frac{20 \hat{k}^{4}-12}{\left(1+\hat{k}^{4}\right)^{2}} \hat{k}^{2} \kappa^{2}-y^{2}\right) \frac{\cos \left(\frac{2 \pi n-y}{\kappa} \hat{k}\right)+\cos \left(\frac{2 \pi n+y}{\kappa} \hat{k}\right)}{2\left(1+\hat{k}^{4}\right)}\right. \\
& \left.+8 y \kappa \hat{k}^{3} \frac{-\sin \left(\frac{2 \pi n-y}{\kappa} \hat{k}\right)+\sin \left(\frac{2 \pi n+y}{\kappa} \hat{k}\right)}{2\left(1+\hat{k}^{4}\right)^{2}}\right] \mathrm{d} \hat{k} \mid
\end{aligned}
$$

for $y=x \kappa$ and $\hat{k}=k \kappa$. Now,

$$
\int_{0}^{\infty}\left|g^{\kappa}(x)-g(x)\right| \mathrm{d} x=\int_{0}^{\frac{\pi}{\kappa}}\left|g^{\kappa}(x)-g(x)\right| \mathrm{d} x+\int_{\frac{\pi}{\kappa}}^{\infty}|g(x)| \mathrm{d} x .
$$

Using $g(x)=\frac{\pi}{2} \mathrm{e}^{-x / \sqrt{2}} \sin \left(\frac{\pi}{4}+\frac{x}{\sqrt{2}}\right)$, the second integral approaches zero as $\kappa \rightarrow \infty$. The first integral becomes

$$
\begin{aligned}
& \int_{0}^{\pi}\left|\sum_{n=1}^{\infty} \frac{1}{(2 \pi n)^{2}} \int_{0}^{\infty}\left[\left(\frac{20 \hat{k}^{4}-12}{\left(1+\hat{k}^{4}\right)^{2}} \hat{k}^{2} \kappa-\frac{y^{2}}{\kappa}\right) \frac{\cos \left(\frac{2 \pi n-y}{\kappa} \hat{k}\right)+\cos \left(\frac{2 \pi n+y}{\kappa} \hat{k}\right)}{2\left(1+\hat{k}^{4}\right)}+8 y \hat{k}^{3} \frac{-\sin \left(\frac{2 \pi n-y}{\kappa} \hat{k}\right)+\sin \left(\frac{2 \pi n+y}{\kappa} \hat{k}\right)}{2\left(1+\hat{k}^{4}\right)^{2}}\right] \mathrm{d} \hat{k}\right| \mathrm{d} y \\
\leq & \sum_{n=1}^{\infty} \frac{1}{(2 \pi n)^{2}} \int_{(2 n-1) \pi}^{(2 n+1) \pi}\left|\int_{0}^{\infty} \cos \left(\frac{y}{\kappa} \hat{k}\right) \frac{10 \hat{k}^{4}-6}{\left(1+\hat{k}^{4}\right)^{3}} \hat{k}^{2} \kappa \mathrm{d} \hat{k}\right|+\left|\int_{0}^{\infty} \cos \left(\frac{y}{\kappa} \hat{k}\right) \frac{\pi^{2}}{2\left(1+\hat{k}^{4}\right) \kappa} \mathrm{d} \hat{k}\right|+\left|\int_{0}^{\infty} \sin \left(\frac{y}{\kappa} \hat{k}\right) \frac{4 \pi \hat{k}^{3}}{\left(1+\hat{k}^{4}\right)^{2}} \mathrm{~d} \hat{k}\right| \mathrm{d} y .
\end{aligned}
$$


The inner integrals in $\hat{k}$ may all be viewed as Fourier coefficients of certain functions at the frequency $\frac{y}{\kappa}$. Since these functions are integrable, their Fourier coefficients decay to zero as the frequency goes to infinity. Hence, all inner integrals uniformly converge to zero as $\kappa \rightarrow \infty$, and so does the complete term.

Acknowledgements. The authors are indebted to Bob Kohn for numerous detailed discussions on the topic.

\section{REFERENCES}

[1] B.P. Athreya, N. Goldenfeld, J.A. Dantzig, M. Greenwood and N. Provatas, Adaptive mesh computation of polycrystalline pattern formation using a renormalization-group reduction of the phase-field crystal model. Phys. Rev. E 76 (2007) 056706.

[2] A.L. Bertozzi, S. Esedoḡlu and A. Gillette, Inpainting of binary images using the Cahn-Hilliard equation. IEEE Trans. Image Process. 16 (2007) 285-291.

[3] M. Cheng and J.A. Warren, An efficient algorithm for solving the phase field crystal model. J. Comput. Phys. 227 (2008) 6241-6248.

[4] K.R. Elder and M. Grant, Modeling elastic and plastic deformations in nonequilibrium processing using phase field crystals. Phys. Rev. E 70 (2004) 051605.

[5] K.R. Elder, M. Katakowski, M. Haataja and M. Grant, Modeling elasticity in crystal growth. Phys. Rev. Lett. 88 (2002) 245701.

[6] K.R. Elder, N. Provatas, J. Berry, P. Stefanovic and M. Grant, Phase-field crystal modeling and classical density functional theory of freezing. Phys. Rev. B $\mathbf{7 5}$ (2007) 064107.

[7] D. Eyre, Unconditionally gradient stable time marching the Cahn-Hilliard equation, in Computational and mathematical models of microstructural evolution, edited by J.W. Bullard, R. Kalia, M. Stoneham and L.Q. Chen. Warrendale, PA, Materials Research Society 53 (1998) 1686-1712.

[8] K. Glasner, A diffuse interface approach to Hele-Shaw flow. Nonlinearity 16 (2003) 49-66.

[9] M. Khenner, A. Averbuch, M. Israeli and M. Nathan, Numerical simulation of grain-boundary grooving by level set method. J. Comput. Phys. 170 (2001) 764-784.

[10] J.N. Lyness and B.J.J. McHugh, On the remainder term in the $N$-dimensional Euler Maclaurin expansion. Numer. Math. 15 (1970) 333-344.

[11] P. Smereka, Semi-implicit level set methods for curvature and surface diffusion motion. J. Sci. Comput. 19 (2003) $439-456$. Special issue in honor of the sixtieth birthday of Stanley Osher.

[12] S.M. Wise, C. Wang and J.S. Lowengrub, An energy-stable and convergent finite-difference scheme for the phase field crystal equation. SIAM J. Numer. Anal. 47 (2009) 2269-2288.

[13] K.-A. Wu and A. Karma, Phase-field crystal modeling of equilibrium bcc-liquid interfaces. Phys. Rev. B 76 (2007) 184107.

[14] K.-A. Wu, M. Plapp and P.W. Voorhees, Controlling crystal symmetries in phase-field crystal models. J. Phys. Condensed Matter 22 (2010) 364102.

[15] K.-A. Wu and P.W. Voorhees, Stress-induced morphological instabilities at the nanoscale examined using the phase field crystal approach. Phys. Rev. B 80 (2009) 125408.

[16] D.-H. Yeon, Z.-F. Huang, K. Elder and K. Thornton, Density-amplitude formulation of the phase-field crystal model for two-phase coexistence in two and three dimensions. Philosophical Magazine 90 (2010) 237-263. 